\title{
The Catalogue of Vascular Plants of the Southern Cone and the Flora of Argentina: their contribution to the World Flora
}

\author{
Fernando O. Zuloaga ${ }^{1,2}$ \& Manuel J. Belgrano ${ }^{1}$
}

\begin{abstract}
The checklist of the vascular plants of the Southern Cone presents updated information of 19,787 taxa and 44,943 synonyms, distributed in 2,679 genera and 318 families. This checklist was prepared, and its permanently updated, using the database "Documenta Florae Australis", with the contribution of numerous researchers and institutions, and constitutes the bases for the Flora of Argentina, currently under preparation. Here we evaluate the current knowledge of vascular plants in the Southern Cone and provide a summary of the ongoing Flora of Argentina, and the significance of these projects for the World Flora on line and to the botanical studies in the region.
\end{abstract}

Key words: Endemism, Flora, South America, Southern Cone.

\begin{abstract}
Resumen
A listagem das plantas vasculares do Cone Sul apresenta 19.787 táxons com 44.943 sinônimos, distribuídos em 2.679 gêneros e 318 famílias. Preparada e continuamene atualizada utilizando o banco de dados "Documenta florae Australis", com a contribuição de vários pesquisadores e instituições, esta lista constitui a base para a Flora da Argentina, atualmente em preparação. Aqui avaliamos o conhecimento atual das plantas vasculares no Cone Sul e fornecemos um resumo sobre o andamento da Flora da Argentina, além da importância destes projetos para a Flora do Mundo Online e para os estudos botânicos na região.
\end{abstract}

Palavras clave: Cono Sur, Endemismo, Flora, Sudamérica.

\section{Introduction}

The Catálogo de las Plantas Vasculares del Cono Sur presents a critical, commented list of the species and infraspecific taxa that occur in the extreme south of the Americas, comprising the totality of Argentina, southern Brazil (Paraná, Santa Catarina and Rio Grande do Sul states), Chile, Paraguay and Uruguay, including the majority of the subtropical and temperate to cold-temperate areas approximately south of $20^{\circ} \mathrm{S}$. The land area is equivalent to $4,708,617 \mathrm{~km}^{2}$, representing $26 \%$ of the total land area of South America (Fig. 1).

This project was preceeded by the Catálogo de Plantas Vasculares de la República Argentina (Zuloaga et al. 1994; Zuloaga \& Morrone 1996, 1999), and started in 2001 at the Instituto de Botánica Darwinion (SI), Buenos Aires, Argentina, in collaboration with the Missouri Botanical
Garden (MO), the Herbarium of the Universidad de Concepción (CONC), Chile, Herbarium of the Facultad de Agronomía (MVFA), Montevideo, Uruguay, the Herbarium of the Facultad de Ciencias Químicas (FCQ), Asunción, Paraguay and the Botany Departament of the Universidade Federal do Rio Grande do Sul (ICN), Porto Alegre, Brazil, involving numerous contributors from different botanical institutions. The resulting critical list of vascular plants from the Southern Cone, or Cono Sur in Spanish, was published in a printed version (Zuloaga et al. 2008), while the digital version is continuously updated and available for public searches through the Instituto Darwinion's webpage $(<\mathrm{http} / / / \mathrm{www}$.darwin.edu. $\mathrm{ar} /$ Proyectos/FloraArgentina/fa.htm $>$ ).

During the year 2008, using as a base the existing Catálogo del Cono Sur and other

\footnotetext{
${ }^{1}$ Instituto de Botánica Darwinion, Labardén 200, Casilla de Correo 22 (B1642HYD), San Isidro, Buenos Aires, Argentina.

${ }^{2}$ Author for correspondence: fzuloaga@darwin.edu.ar
} 


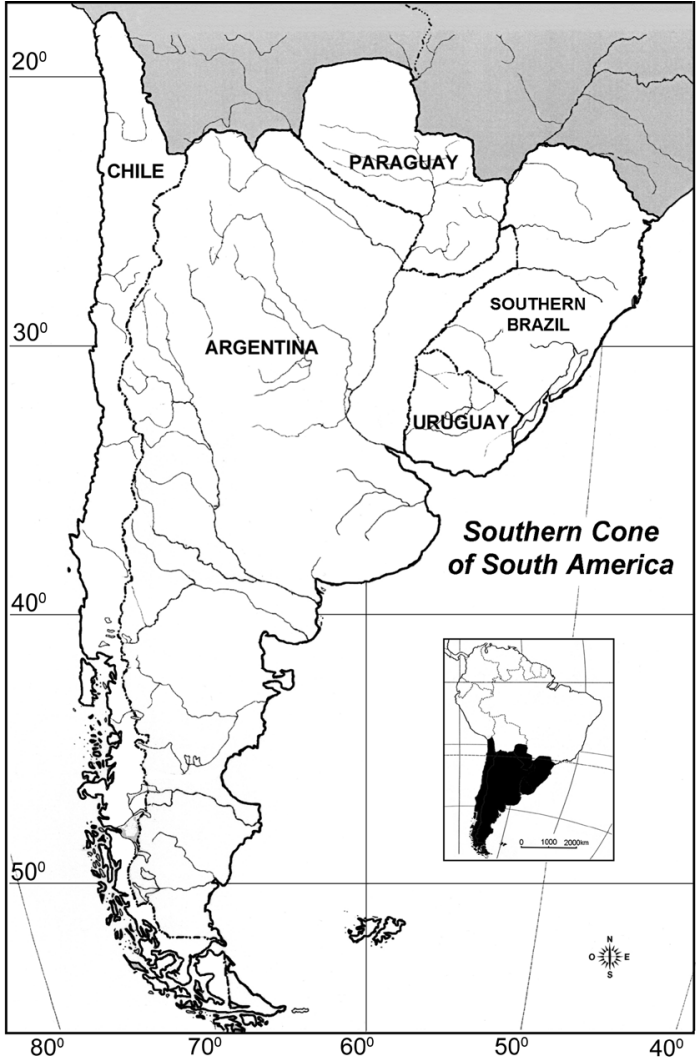

Figure 1 - Study area, the Southern Cone of South America (Argentina, Southern Brazil, Chile, Paraguay, and Uruguay).

previous projects carried out in Argentina (Floras of Buenos Aires, Chaqueña, Entre Ríos, Jujuy, Patagonia, San Juan) and also the Proflora project (Hunziker 1994-2001; Anton \& Zuloaga 20012005), the production and publication of a new Flora vascular de la República Argentina (from now on Flora of Argentina) begun. This work was planned to comprise 20 volumes and is currently underway, with an electronic version available at $(<$ http://www.floraargentina.edu.ar $>)$. The web version incorporates additional information such as distribution maps, photographs of live plants in the field and complete list of examined specimens. To date, the published volumes of Flora of Argentina include Poaceae (Zuloaga et al. 2012), Asteraceae (parts 1, tribes Anthemideae to Gnaphalieae and 3, tribes Senecioneae and Vernonieae) (Zuloaga et al. 2014a, b), Brassicaceae (Anton \& Zuloaga 2012a), Solanaceae (Zuloaga et al. 2013) and Verbenaceae (Anton \& Zuloaga 2012b); the volumes regarding Basal Dicotyledoneae (published in September 2015) and Asteraceae (volume 7(2) is in press).
This contribution updates the floristic information found in the Catálogo de las plantas vasculares del Cono Sur (Zuloaga et al. 2008), particularly concerning data from Argentina, as the ongoing preparation of the Flora of Argentina, directed by A. M. Anton (CORD) \& F. O. Zuloaga (SI), including analyses and floristic comments that will lead to future systematic works in the region, and to the integration of these projects into the World Flora online by 2020 .

\section{Methodology}

The present work used the database "Documenta Florae Australis" (from now on referred as DFA), developed and maintained at the Instituto Darwinion during the last 20 years. Such relational database integrates scientific name of the accepted taxa with their synonyms, indicates original publication data, presence and distribution in each country of the South American Southern Cone (discriminating by regions, provinces or states, departments or municipalities), status (if the taxon is endemic, native or introduced), habit and lifecycle, including also bibliography and vouchers, line drawings and field photographs. A complete inventory of the vascular plants cited for the Southern Cone can be obtained from DFA. As soon as new taxonomic and systematic treatments are published, DFA data is updated and its records are increased daily by the contribution of numerous registered users. Also, more than 15 institutions are digitalising their collections and these are continuously uploaded into this system. Today, DFA has 66,784 scientific names recorded for the Southern Cone, of which 19,787 are accepted and the remaining 44,943 are synonyms. The number of herbarium specimens linked to the system is 442,155 , the bibliography records amount to 10,005 , while 12,554 names of taxonomists, collectors or botanical authorities, close to 9,000 line art illustrations and 35,000 pictures of plants in the field, amongst other items, are included in DFA. This database is open and available for consultation and represents a valuable resource for improving knowledge, management and conservation of the flora of Southern South America.

Angiosperm families from the Southern Cone were organized according to the Angiosperm Phylogeny Group available at ( $<$ http://www.mobot. org/MOBOT/research/APweb/>), with a few punctual exceptions where the specialists preferred to maintain the traditional classification, such as the Chenopodiaceae, that remains separate from the 
Amaranthaceae. For the Ferns and allies the system of R. M. Tryon \& A. F. Tryon (1982) was followed, with subsequent modifications and updates (in the classification of families and genera).

Distributional analysis of taxa followed the Biogeographical scheme proposed by Josse et al. (2003) (Fig. 2) with slight modifications.

\section{Results and Discussion}

A figure of 19,787 taxa, distribuited in 18,139 species, 2,679 genera and 318 families is currently recorded for the Southern Cone, 7,787 species are endemic to the region (Tab. 1). The Angiosperms comprise $95.40 \%$ of the vascular flora $(70.68 \%$ Dicots and $24,72 \%$ Monocots) including the majority of the endemic species from the Southern Cone (7,630 species, or $85.08 \%$ of the total).

The significant difference found between the present results and those published in the Catálogo del Cono Sur (Zuloaga et al. 2008: xxxviii), considering that a short period of time has elapsed between those milestones, points out the importance of keeping the floristic and taxonomic information available online and permanently updated. The total of families in 2008 was 308, with 2,586 genera and 17,693 species, of which 7,691 were endemic. The increase in family numbers was 10 while the genera increased by 93 , with 446 additional species; of these, 96 more were endemic. There are several possible causes to explain this variation: description of new taxa, record of new occurrences for the area or adjustment of taxonomic circumscription at all levels, often as a result of new taxonomic and phylogenetic research. Therefore the species number accepted within a certain genus may decrease when new synonymies are proposed, as seen in genus Phyla L. (O’Leary \& Múlgura 2012) or it may increase through the description of new species, as was the case with Chusquea Kunth, through the description of Chusquea egluma Guerreiro \& Rúgolo and Chusquea floribunda Guerreiro \&

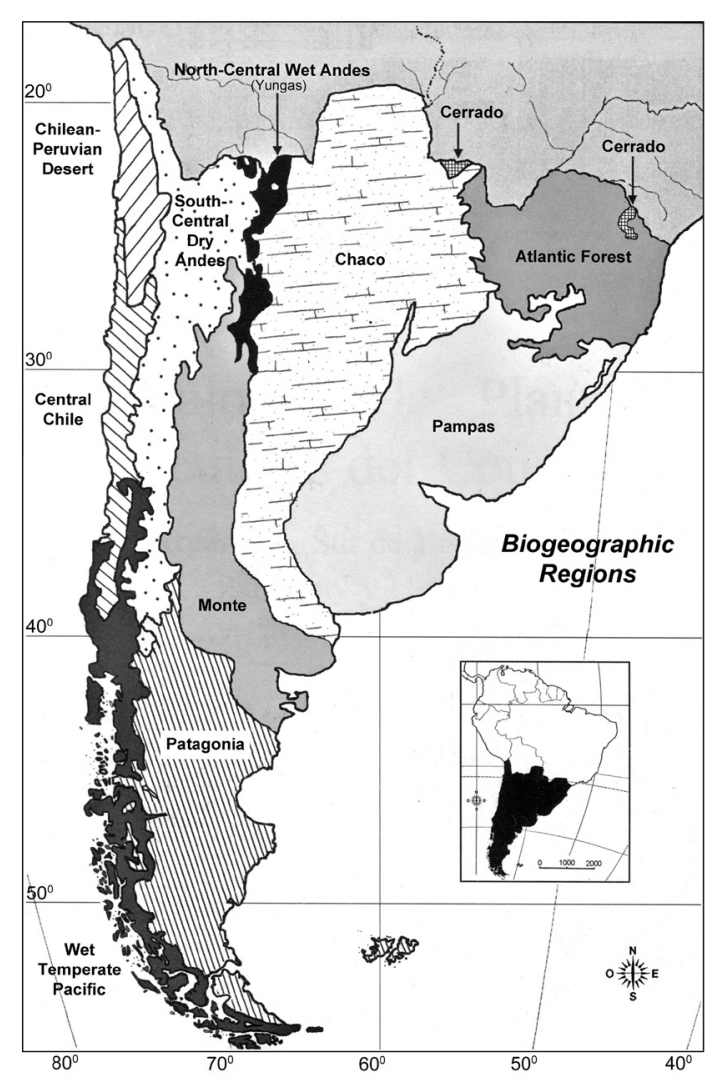

Figure 2-Biogeographic regions of the Southern Cone of South America.

Rúgolo in 2013 (Guerreiro \& Rúgolo de Agrasar 2013). The number of genera may increase through segregation of a group of species to another genus, as seen in 2014 for Panicum validum Mez that was split from Panicum L. and placed within the new genus Osvaldoa J.R. Grande (Grande Allende 2014), causing an increase in the number of genera in the Poaceae. The number of families may also decrease, as it is the case of the Nolanaceae and Sclerophylacaceae, nowadays considered as part of the Solanaceae.

Table 1 - Southern Cone, summary of the taxa included in the catalogue.

\begin{tabular}{lcccccc}
\hline Group & Families & Genera & Species & \% Species & Endemics & \% Endemics \\
\hline Pteridophyta & 36 & 124 & 804 & 4,43 & 152 & 18,91 \\
Gymnospermae & 5 & 12 & 30 & 0,17 & 5 & 16,67 \\
Dicotyledoneae & 220 & 1929 & 12821 & 70,68 & 5867 & 45,76 \\
Monocotyledoneae & 57 & 614 & 4484 & 24,72 & 1763 & 39,32 \\
\hline Total & 318 & 2679 & 18139 & & 7787 & \\
\hline
\end{tabular}


Table 2 - Argentina, summary of the taxa recorded for the country.

\begin{tabular}{lcccccc}
\hline Group & Families & Genera & Species & \% Species & Endemics & \%Endemics \\
\hline Pteridophyta & 34 & 97 & 390 & 3,90 & 16 & 4,10 \\
Gymnospermae & 5 & 12 & 26 & 0,26 & 1 & 3,85 \\
Dicotyledoneae & 194 & 1519 & 7162 & 71,58 & 1383 & 19,31 \\
Monocotyledoneae & 50 & 462 & 2428 & 24,27 & 349 & 14,37 \\
\hline Total & 283 & 2090 & 10006 & & 1749 & \\
\hline
\end{tabular}

For the Flora of Argentina, there is a current record of 10,944 taxa distributed in 10,006 species, 2,090 genera and 283 families, with a total of 1,749 endemic species (Tab. 2) representing $17.48 \%$ of the country's flora.

\section{Plant diversity at family level}

The largest family in terms of species number in the Southern Cone is Asteraceae (2,523 species), followed by Poaceae $(1,535)$, Fabaceae $(1,365)$, Orchidaceae $(1,042)$, Solanaceae (546), Cyperaceae (485), Euphorbiaceae (447), Malvaceae (408), Cactaceae (406) and Apocynaceae (333). These families comprise $50 \%$ of the species found in the area. Contrastingly, 202 families are represented by 15 or less species, of which 59 have a single species in the area. A complete list of the families verified for the Southern Cone is presented in Table 3, indicating the genus, species and endemic species number for each, together with the percentage of endemic species in relation to the total number of species in the family.

Argentina has a similar representation at family level to what is found in the Southern Cone $(55.16 \%$ of the species from the Southern Cone are present in Argentina); the top ten families are Asteraceae (1,502 species), Poaceae $(1,170)$, Fabaceae (760), Solanaceae (322), Cyperaceae (314), Orchidaceae (281), Cactaceae (240), Malvaceae (233), Brassicaceae (229) and Euphorbiaceae (218); 202 families with 15 or less species among which 68 have a single representative in the country (Tab. 4).

Plant diversity at genus level

Among the 2,679 genera found in the Southern Cone, the largest one is Senecio L. (Asteraceae, 415 species), followed by Solanum L. (Solanaceae, 213), Baccharis L. (Asteraceae, 208), Adesmia DC. (Fabaceae, 199), Mimosa
L. (Fabaceae, 169), Croton L. (Euphorbiaceae, 126), Oxalis L. (Oxalidaceae, 124), Eugenia L. (Myrtaceae, 122), Carex L. (Cyperaceae, 110), Paspalum L. (Poaceae, 109), Viola L. (Violaceae, 107) and Sisyrinchium L. (Iridaceae, 103). The remaining genera have 100 or less species recorded for the region.

Particularly, for the Flora of Argentina, the richest genus in number of species is Senecio (with 260 species), followed by Solanum (126), Adesmia (102), Baccharis (101), Paspalum (77), Oxalis (71), Astragalus L. (69), Mimosa (53), Viola (53), Valeriana L. (48), Polygala L. (44) and Calceolaria L. (41). In the other side of the spectrum, 949 genera ( $45.40 \%$ of the total) are represented by a single species in Argentina.

\section{Endemism}

A species or taxon is considered 'endemic' in the present work when its distribution is known only for the area of this project. Thus, for the Southern Cone, there are eight endemic families $(3.25 \%$ of the total number of families): Francoaceae, Gomortegaceae and Lactoridaceae, known only in Chile, Halophytaceae, exclusive from Argentina and Aextoxicaceae, Misodendraceae, Philesiaceae and Tribelaceae, shared between Argentina and Chile. The number of endemic genera for the region is 241 ( $9 \%$ of the total genera) and the family with the largest number of endemic genera is Asteraceae, with 46 endemic genera, followed by Amaryllidaceae (16), Brassicaceae (13), Apiaceae (11), Cactaceae (10), Solanaceae (9), Poaceae (9), Alliaceae (8) and Fabaceae (6). Table 5 details the endemic genera with species number and their presence in each country. The total of endemic species in the Southern Cone is 7,787, representing $42,93 \%$ of the total plant species found in the region.

Meanwhile, for the Flora of Argentina, the only endemic family is the monotypic 
Table 3 - Southern Cone, number of families, genera, species, and endemic species.

\begin{tabular}{|c|c|c|c|c|c|}
\hline Group & Family & Genera & Species & Endemics & $\%$ Endemism \\
\hline Dicotyledoneae & Acanthaceae & 20 & 124 & 28 & 22,6 \\
\hline Dicotyledoneae & Aceraceae & 1 & 2 & & \\
\hline Dicotyledoneae & Achariaceae & 1 & 1 & & \\
\hline Dicotyledoneae & Achatocarpaceae & 1 & 5 & 2 & 40,0 \\
\hline Dicotyledoneae & Adoxaceae & 2 & 3 & & \\
\hline Dicotyledoneae & Aextoxicaceae & 1 & 1 & 1 & 100,0 \\
\hline Monocotyledoneae & Agapanthaceae & 1 & 1 & & \\
\hline Monocotyledoneae & Agavaceae & 2 & 3 & & \\
\hline Dicotyledoneae & Aizoaceae & 7 & 20 & 9 & 45,0 \\
\hline Monocotyledoneae & Alismataceae & 4 & 18 & 3 & 16,7 \\
\hline Monocotyledoneae & Alstroemeriaceae & 3 & 56 & 42 & 75,0 \\
\hline Dicotyledoneae & Amaranthaceae & 12 & 140 & 43 & 30,7 \\
\hline Monocotyledoneae & Amaryllidaceae & 29 & 294 & 247 & 84,0 \\
\hline Dicotyledoneae & Anacampserotaceae & 2 & 3 & 3 & 100,0 \\
\hline Dicotyledoneae & Anacardiaceae & 10 & 49 & 18 & 36,7 \\
\hline Pteridophyta & Anemiaceae & 1 & 14 & 1 & 7,1 \\
\hline Dicotyledoneae & Annonaceae & 5 & 28 & 6 & 21,4 \\
\hline Monocotyledoneae & Anthericaceae & 2 & 8 & 3 & 37,5 \\
\hline Dicotyledoneae & Apiaceae & 48 & 225 & 133 & 59,1 \\
\hline Dicotyledoneae & Apocynaceae & 61 & 333 & 122 & 36,6 \\
\hline Monocotyledoneae & Aponogetonaceae & 1 & 1 & & \\
\hline Dicotyledoneae & Aquifoliaceae & 1 & 12 & 8 & 66,7 \\
\hline Monocotyledoneae & Araceae & 21 & 58 & 9 & 15,5 \\
\hline Dicotyledoneae & Araliaceae & 6 & 13 & 2 & 15,4 \\
\hline Gymnospermae & Araucariaceae & 1 & 2 & 1 & 50,0 \\
\hline Monocotyledoneae & Arecaceae & 15 & 50 & 22 & 44,0 \\
\hline Dicotyledoneae & Aristolochiaceae & 1 & 34 & 12 & 35,3 \\
\hline Monocotyledoneae & Asparagaceae & 1 & 5 & & \\
\hline Monocotyledoneae & Asphodelaceae & 1 & 1 & & \\
\hline Pteridophyta & Aspleniaceae & 2 & 65 & 7 & 10,8 \\
\hline Monocotyledoneae & Asteliaceae & 1 & 1 & 1 & 100,0 \\
\hline Dicotyledoneae & Asteraceae & 327 & 2523 & 1444 & 57,2 \\
\hline Pteridophyta & Athyriaceae & 3 & 19 & & \\
\hline Dicotyledoneae & Avicenniaceae & 1 & 1 & & \\
\hline Pteridophyta & Azollaceae & 1 & 2 & & \\
\hline Dicotyledoneae & Balanophoraceae & 4 & 5 & & \\
\hline Dicotyledoneae & Balsaminaceae & 1 & 3 & & \\
\hline Dicotyledoneae & Basellaceae & 2 & 4 & & \\
\hline Dicotyledoneae & Begoniaceae & 1 & 55 & 36 & 65,5 \\
\hline Dicotyledoneae & Berberidaceae & 1 & 34 & 31 & 91,2 \\
\hline Dicotyledoneae & Berberidopsidaceae & 1 & 1 & 1 & 100,0 \\
\hline
\end{tabular}




\begin{tabular}{|c|c|c|c|c|c|}
\hline Group & Family & Genera & Species & Endemics & $\%$ Endemism \\
\hline Dicotyledoneae & Betulaceae & 2 & 3 & & \\
\hline Dicotyledoneae & Bignoniaceae & 29 & 124 & 19 & 15,3 \\
\hline Dicotyledoneae & Bixaceae & 1 & 1 & & \\
\hline Pteridophyta & Blechnaceae & 2 & 36 & 11 & 30,6 \\
\hline Dicotyledoneae & Bombacaceae & 5 & 12 & & \\
\hline Dicotyledoneae & Boraginaceae & 31 & 214 & 106 & 49,5 \\
\hline Dicotyledoneae & Brassicaceae & 71 & 302 & 180 & 59,6 \\
\hline Monocotyledoneae & Bromeliaceae & 24 & 302 & 147 & 48,7 \\
\hline Monocotyledoneae & Burmanniaceae & 6 & 9 & & \\
\hline Dicotyledoneae & Burseraceae & 2 & 3 & 1 & 33,3 \\
\hline Dicotyledoneae & Cabombaceae & 1 & 2 & & \\
\hline Dicotyledoneae & Cactaceae & 55 & 406 & 290 & 71,4 \\
\hline Dicotyledoneae & Calceolariaceae & 2 & 88 & 68 & 77,3 \\
\hline Dicotyledoneae & Callitrichaceae & 1 & 9 & 4 & 44,4 \\
\hline Dicotyledoneae & Calyceraceae & 6 & 48 & 43 & 89,6 \\
\hline Dicotyledoneae & Campanulaceae & 10 & 51 & 17 & 33,3 \\
\hline Dicotyledoneae & Canellaceae & 2 & 2 & 1 & 50,0 \\
\hline Dicotyledoneae & Cannabaceae & 1 & 2 & & \\
\hline Monocotyledoneae & Cannaceae & 1 & 5 & 1 & 20,0 \\
\hline Dicotyledoneae & Capparaceae & 10 & 25 & 4 & 16,0 \\
\hline Dicotyledoneae & Caprifoliaceae & 2 & 2 & & \\
\hline Dicotyledoneae & Cardiopteridaceae & 1 & 6 & 1 & 16,7 \\
\hline Dicotyledoneae & Caricaceae & 2 & 6 & 1 & 16,7 \\
\hline Dicotyledoneae & Caryocaraceae & 1 & 1 & & \\
\hline Dicotyledoneae & Caryophyllaceae & 27 & 174 & 83 & 47,7 \\
\hline Dicotyledoneae & Cecropiaceae & 3 & 4 & & \\
\hline Dicotyledoneae & Celastraceae & 13 & 33 & 8 & 24,2 \\
\hline Dicotyledoneae & Celtidaceae & 2 & 5 & & \\
\hline Monocotyledoneae & Centrolepidaceae & 1 & 1 & 1 & 100,0 \\
\hline Dicotyledoneae & Ceratophyllaceae & 1 & 2 & & \\
\hline Dicotyledoneae & Cervantesiaceae & 2 & 3 & 1 & 33,3 \\
\hline Dicotyledoneae & Chenopodiaceae & 17 & 125 & 60 & 48,0 \\
\hline Dicotyledoneae & Chloranthaceae & 1 & 1 & & \\
\hline Dicotyledoneae & Chrysobalanaceae & 4 & 6 & & \\
\hline Dicotyledoneae & Cistaceae & 1 & 1 & 1 & 100,0 \\
\hline Dicotyledoneae & Clethraceae & 1 & 2 & 1 & 50,0 \\
\hline Dicotyledoneae & Clusiaceae & 5 & 7 & & \\
\hline Dicotyledoneae & Cochlospermaceae & 1 & 2 & & \\
\hline Dicotyledoneae & Combretaceae & 5 & 16 & 4 & 25,0 \\
\hline Monocotyledoneae & Commelinaceae & 8 & 29 & 2 & 6,9 \\
\hline Dicotyledoneae & Connaraceae & 2 & 2 & & \\
\hline Dicotyledoneae & Convolvulaceae & 14 & 198 & 77 & 38,9 \\
\hline Dicotyledoneae & Coriariaceae & 1 & 1 & 1 & 100,0 \\
\hline Monocotyledoneae & Corsiaceae & 1 & 1 & 1 & 100,0 \\
\hline
\end{tabular}




\begin{tabular}{|c|c|c|c|c|c|}
\hline Group & Family & Genera & Species & Endemics & $\%$ Endemism \\
\hline Monocotyledoneae & Costaceae & 1 & 3 & & \\
\hline Dicotyledoneae & Crassulaceae & 4 & 17 & 5 & 29,4 \\
\hline Dicotyledoneae & Cucurbitaceae & 24 & 79 & 16 & 20,3 \\
\hline Dicotyledoneae & Cunoniaceae & 3 & 9 & 2 & 22,2 \\
\hline Gymnospermae & Cupressaceae & 4 & 4 & 3 & 75,0 \\
\hline Pteridophyta & Cyatheaceae & 3 & 17 & & \\
\hline Monocotyledoneae & Cyclanthaceae & 1 & 2 & 1 & 50,0 \\
\hline Monocotyledoneae & Cymodoceaceae & 1 & 1 & & \\
\hline Monocotyledoneae & Cyperaceae & 33 & 485 & 162 & 33,4 \\
\hline Pteridophyta & Dennstaedtiaceae & 5 & 14 & 1 & 7,1 \\
\hline Dicotyledoneae & Desfontainiaceae & 1 & 1 & & \\
\hline Pteridophyta & Dicksoniaceae & 3 & 5 & 3 & 60,0 \\
\hline Pteridophyta & Didymochlaenaceae & 1 & 1 & & \\
\hline Dicotyledoneae & Dilleniaceae & 4 & 11 & & \\
\hline Monocotyledoneae & Dioscoreaceae & 1 & 85 & 53 & 62,4 \\
\hline Dicotyledoneae & Dipsacaceae & 3 & 5 & & \\
\hline Dicotyledoneae & Donatiaceae & 1 & 1 & 1 & 100,0 \\
\hline Dicotyledoneae & Droseraceae & 1 & 6 & 1 & 16,7 \\
\hline Pteridophyta & Dryopteridaceae & 15 & 120 & 32 & 26,7 \\
\hline Dicotyledoneae & Ebenaceae & 1 & 3 & 1 & 33,3 \\
\hline Dicotyledoneae & Elaeagnaceae & 1 & 1 & & \\
\hline Dicotyledoneae & Elaeocarpaceae & 4 & 10 & 4 & 40,0 \\
\hline Dicotyledoneae & Elatinaceae & 1 & 2 & 1 & 50,0 \\
\hline Dicotyledoneae & Empetraceae & 1 & 1 & & \\
\hline Dicotyledoneae & Epacridaceae & 1 & 1 & 1 & 100,0 \\
\hline Gymnospermae & Ephedraceae & 1 & 12 & 8 & 66,7 \\
\hline Pteridophyta & Equisetaceae & 1 & 2 & & \\
\hline Dicotyledoneae & Eremolepidaceae & 3 & 3 & 2 & 66,7 \\
\hline Dicotyledoneae & Ericaceae & 5 & 39 & 21 & 53,8 \\
\hline Monocotyledoneae & Eriocaulaceae & 5 & 40 & 20 & 50,0 \\
\hline Dicotyledoneae & Erythroxylaceae & 1 & 19 & 4 & 21,1 \\
\hline Dicotyledoneae & Escalloniaceae & 2 & 29 & 18 & 62,1 \\
\hline Dicotyledoneae & Eucryphiaceae & 1 & 2 & 2 & 100,0 \\
\hline Dicotyledoneae & Euphorbiaceae & 40 & 447 & 205 & 45,9 \\
\hline Dicotyledoneae & Fabaceae & 160 & 1365 & 624 & 45,7 \\
\hline Dicotyledoneae & Francoaceae & 2 & 2 & 2 & 100,0 \\
\hline Dicotyledoneae & Frankeniaceae & 1 & 10 & 6 & 60,0 \\
\hline Dicotyledoneae & Fumariaceae & 1 & 7 & & \\
\hline Dicotyledoneae & Gentianaceae & 15 & 57 & 21 & 36,8 \\
\hline Dicotyledoneae & Geraniaceae & 2 & 26 & 10 & 38,5 \\
\hline Dicotyledoneae & Gesneriaceae & 10 & 48 & 18 & 37,5 \\
\hline Pteridophyta & Gleicheniaceae & 3 & 18 & 4 & 22,2 \\
\hline Dicotyledoneae & Gomortegaceae & 1 & 1 & 1 & 100,0 \\
\hline
\end{tabular}




\begin{tabular}{|c|c|c|c|c|c|}
\hline Group & Family & Genera & Species & Endemics & $\%$ Endemism \\
\hline Dicotyledoneae & Goodeniaceae & 2 & 2 & & \\
\hline Dicotyledoneae & Griseliniaceae & 1 & 5 & 4 & 80,0 \\
\hline Dicotyledoneae & Grossulariaceae & 1 & 12 & 10 & 83,3 \\
\hline Dicotyledoneae & Gunneraceae & 1 & 11 & 7 & 63,6 \\
\hline Dicotyledoneae & Halophytaceae & 1 & 1 & 1 & 100,0 \\
\hline Dicotyledoneae & Haloragaceae & 4 & 6 & 2 & 33,3 \\
\hline Monocotyledoneae & Heliconiaceae & 1 & 6 & & \\
\hline Monocotyledoneae & Hemerocallidaceae & 1 & 1 & & \\
\hline Monocotyledoneae & Herreriaceae & 2 & 6 & 5 & 83,3 \\
\hline Dicotyledoneae & Hippuridaceae & 1 & 1 & & \\
\hline Dicotyledoneae & Humiriaceae & 1 & 1 & & \\
\hline Monocotyledoneae & Hyacinthaceae & 1 & 5 & 1 & 20,0 \\
\hline Dicotyledoneae & Hydnoraceae & 1 & 2 & & \\
\hline Dicotyledoneae & Hydrangeaceae & 1 & 1 & 1 & 100,0 \\
\hline Monocotyledoneae & Hydrocharitaceae & 5 & 7 & & \\
\hline Dicotyledoneae & Hydroleaceae & 1 & 2 & & \\
\hline Pteridophyta & Hymenophyllaceae & 6 & 68 & 23 & 33,8 \\
\hline Dicotyledoneae & Hypericaceae & 1 & 28 & 14 & 50,0 \\
\hline Monocotyledoneae & Hypoxidaceae & 3 & 5 & 1 & 20,0 \\
\hline Dicotyledoneae & Icacinaceae & 1 & 1 & 1 & 100,0 \\
\hline Monocotyledoneae & Iridaceae & 27 & 220 & 148 & 67,3 \\
\hline Pteridophyta & Isoëtaceae & 1 & 20 & 15 & 75,0 \\
\hline Dicotyledoneae & Juglandaceae & 2 & 2 & & \\
\hline Monocotyledoneae & Juncaceae & 7 & 64 & 26 & 40,6 \\
\hline Monocotyledoneae & Juncaginaceae & 3 & 5 & 1 & 20,0 \\
\hline Dicotyledoneae & Krameriaceae & 1 & 3 & 1 & 33,3 \\
\hline Dicotyledoneae & Lacistemataceae & 1 & 3 & & \\
\hline Dicotyledoneae & Lactoridaceae & 1 & 1 & 1 & 100,0 \\
\hline Dicotyledoneae & Lamiaceae & 42 & 220 & 78 & 35,5 \\
\hline Dicotyledoneae & Lardizabalaceae & 2 & 2 & 2 & 100,0 \\
\hline Dicotyledoneae & Lauraceae & 11 & 76 & 9 & 11,8 \\
\hline Monocotyledoneae & Laxmanniaceae & 1 & 2 & 2 & 100,0 \\
\hline Dicotyledoneae & Lecythidaceae & 1 & 1 & & \\
\hline Dicotyledoneae & Ledocarpaceae & 1 & 9 & 6 & 66,7 \\
\hline Monocotyledoneae & Lemnaceae & 5 & 11 & & \\
\hline Dicotyledoneae & Lentibulariaceae & 3 & 25 & 2 & 8,0 \\
\hline Monocotyledoneae & Limnocharitaceae & 2 & 6 & & \\
\hline Dicotyledoneae & Linaceae & 2 & 17 & 5 & 29,4 \\
\hline Dicotyledoneae & Linderniaceae & 2 & 3 & & \\
\hline Pteridophyta & Lindsaeaceae & 1 & 10 & & \\
\hline Dicotyledoneae & Loasaceae & 7 & 69 & 49 & 71,0 \\
\hline Dicotyledoneae & Loganiaceae & 2 & 29 & 12 & 41,4 \\
\hline Pteridophyta & Lomariopsidaceae & 2 & 2 & & \\
\hline
\end{tabular}




\begin{tabular}{|c|c|c|c|c|c|}
\hline Group & Family & Genera & Species & Endemics & $\%$ Endemism \\
\hline Dicotyledoneae & Loranthaceae & 7 & 12 & 6 & 50,0 \\
\hline Monocotyledoneae & Luzuriagaceae & 1 & 3 & 3 & 100,0 \\
\hline Pteridophyta & Lycopodiaceae & 9 & 37 & 13 & 35,1 \\
\hline Pteridophyta & Lygodiaceae & 1 & 2 & & \\
\hline Dicotyledoneae & Lythraceae & 8 & 64 & 16 & 25,0 \\
\hline Dicotyledoneae & Malesherbiaceae & 1 & 16 & 15 & 93,8 \\
\hline Dicotyledoneae & Malpighiaceae & 31 & 116 & 23 & 19,8 \\
\hline Dicotyledoneae & Malvaceae & 45 & 408 & 173 & 42,4 \\
\hline Monocotyledoneae & Marantaceae & 6 & 13 & 2 & 15,4 \\
\hline Pteridophyta & Marattiaceae & 3 & 5 & & \\
\hline Dicotyledoneae & Marcgraviaceae & 2 & 2 & & \\
\hline Pteridophyta & Marsileaceae & 3 & 5 & 1 & 20,0 \\
\hline Dicotyledoneae & Martyniaceae & 2 & 4 & 2 & 50,0 \\
\hline Monocotyledoneae & Mayacaceae & 1 & 2 & & \\
\hline Dicotyledoneae & Melastomataceae & 26 & 225 & 43 & 19,1 \\
\hline Dicotyledoneae & Meliaceae & 5 & 22 & 2 & 9,1 \\
\hline Dicotyledoneae & Menispermaceae & 5 & 15 & 1 & 6,7 \\
\hline Dicotyledoneae & Menyanthaceae & 1 & 2 & & \\
\hline Dicotyledoneae & Microteaceae & 1 & 5 & & \\
\hline Dicotyledoneae & Misodendraceae & 1 & 8 & 8 & 100,0 \\
\hline Dicotyledoneae & Molluginaceae & 2 & 3 & & \\
\hline Dicotyledoneae & Monimiaceae & 4 & 21 & 5 & 23,8 \\
\hline Dicotyledoneae & Montiaceae & 8 & 58 & 49 & 84,5 \\
\hline Dicotyledoneae & Moraceae & 8 & 31 & 1 & 3,2 \\
\hline Dicotyledoneae & Muntingiaceae & 1 & 1 & & \\
\hline Dicotyledoneae & Myoporaceae & 1 & 1 & & \\
\hline Dicotyledoneae & Myricaceae & 1 & 2 & & \\
\hline Dicotyledoneae & Myristicaceae & 1 & 1 & & \\
\hline Dicotyledoneae & Myrsinaceae & 3 & 18 & 3 & 16,7 \\
\hline Dicotyledoneae & Myrtaceae & 28 & 313 & 139 & 44,4 \\
\hline Monocotyledoneae & Najadaceae & 1 & 3 & & \\
\hline Dicotyledoneae & Nanodeaceae & 1 & 1 & 1 & 100,0 \\
\hline Pteridophyta & Nephrolepidaceae & 1 & 6 & & \\
\hline Dicotyledoneae & Nolanaceae & 1 & 50 & 45 & 90,0 \\
\hline Dicotyledoneae & Nothofagaceae & 3 & 10 & 10 & 100,0 \\
\hline Dicotyledoneae & Nyctaginaceae & 10 & 37 & 7 & 18,9 \\
\hline Dicotyledoneae & Nymphaeaceae & 3 & 10 & & \\
\hline Dicotyledoneae & Ochnaceae & 2 & 7 & & \\
\hline Dicotyledoneae & Olacaceae & 1 & 2 & & \\
\hline Dicotyledoneae & Oleaceae & 6 & 17 & 2 & 11,8 \\
\hline Pteridophyta & Oleandraceae & 1 & 1 & 1 & 100,0 \\
\hline Dicotyledoneae & Onagraceae & 7 & 95 & 30 & 31,6 \\
\hline Pteridophyta & Ophioglossaceae & 2 & 12 & 2 & 16,7 \\
\hline
\end{tabular}




\begin{tabular}{|c|c|c|c|c|c|}
\hline Group & Family & Genera & Species & Endemics & $\%$ Endemism \\
\hline Dicotyledoneae & Opiliaceae & 1 & 2 & & \\
\hline Monocotyledoneae & Orchidaceae & 158 & 1042 & 294 & 28,2 \\
\hline Dicotyledoneae & Orobanchaceae & 11 & 59 & 24 & 40,7 \\
\hline Pteridophyta & Osmundaceae & 2 & 3 & & \\
\hline Dicotyledoneae & Oxalidaceae & 2 & 126 & 73 & 57,9 \\
\hline Dicotyledoneae & Papaveraceae & 6 & 14 & 2 & 14,3 \\
\hline Pteridophyta & Parkeriaceae & 1 & 1 & & \\
\hline Dicotyledoneae & Parnassiaceae & 1 & 1 & & \\
\hline Dicotyledoneae & Passifloraceae & 1 & 43 & 3 & 7,0 \\
\hline Dicotyledoneae & Pentaphylacaceae & 1 & 2 & & \\
\hline Monocotyledoneae & Philesiaceae & 2 & 2 & 2 & 100,0 \\
\hline Dicotyledoneae & Phrymaceae & 3 & 9 & 6 & 66,7 \\
\hline Dicotyledoneae & Phyllanthaceae & 5 & 26 & 3 & 11,5 \\
\hline Dicotyledoneae & Phytolaccaceae & 10 & 22 & 6 & 27,3 \\
\hline Dicotyledoneae & Picrodendraceae & 1 & 1 & & \\
\hline Gymnospermae & Pinaceae & 2 & 4 & & \\
\hline Dicotyledoneae & Piperaceae & 4 & 136 & 34 & 25,0 \\
\hline Dicotyledoneae & Plantaginaceae & 28 & 154 & 63 & 40,9 \\
\hline Dicotyledoneae & Plumbaginaceae & 4 & 6 & 4 & 66,7 \\
\hline Monocotyledoneae & Poaceae & 201 & 1535 & 542 & 35,3 \\
\hline Gymnospermae & Podocarpaceae & 4 & 8 & 5 & 62,5 \\
\hline Dicotyledoneae & Podostemaceae & 10 & 19 & 8 & 42,1 \\
\hline Dicotyledoneae & Polemoniaceae & 9 & 13 & 4 & 30,8 \\
\hline Dicotyledoneae & Polygalaceae & 6 & 106 & 50 & 47,2 \\
\hline Dicotyledoneae & Polygonaceae & 15 & 95 & 36 & 37,9 \\
\hline Pteridophyta & Polypodiaceae & 17 & 88 & 5 & 5,7 \\
\hline Monocotyledoneae & Pontederiaceae & 3 & 10 & & \\
\hline Dicotyledoneae & Portulacaceae & 2 & 31 & 17 & 54,8 \\
\hline Monocotyledoneae & Potamogetonaceae & 2 & 12 & 2 & 16,7 \\
\hline Dicotyledoneae & Primulaceae & 7 & 12 & 4 & 33,3 \\
\hline Dicotyledoneae & Proteaceae & 7 & 14 & 6 & 42,9 \\
\hline Pteridophyta & Psilotaceae & 1 & 1 & & \\
\hline Pteridophyta & Pteridaceae & 18 & 127 & 22 & 17,3 \\
\hline Dicotyledoneae & Quiinaceae & 1 & 1 & & \\
\hline Dicotyledoneae & Quillajaceae & 1 & 2 & 1 & 50,0 \\
\hline Dicotyledoneae & Rafflesiaceae & 1 & 5 & 1 & 20,0 \\
\hline Dicotyledoneae & Ranunculaceae & 12 & 65 & 31 & 47,7 \\
\hline Dicotyledoneae & Resedaceae & 1 & 4 & & \\
\hline Monocotyledoneae & Restionaceae & 1 & 1 & 1 & 100,0 \\
\hline Dicotyledoneae & Rhamnaceae & 16 & 39 & 20 & 51,3 \\
\hline Dicotyledoneae & Rhizophoraceae & 1 & 1 & & \\
\hline Dicotyledoneae & Rosaceae & 22 & 125 & 51 & 40,8 \\
\hline Dicotyledoneae & Rubiaceae & 62 & 293 & 87 & 29,7 \\
\hline
\end{tabular}




\begin{tabular}{|c|c|c|c|c|c|}
\hline Group & Family & Genera & Species & Endemics & \% Endemism \\
\hline Monocotyledoneae & Ruppiaceae & 1 & 3 & & \\
\hline Monocotyledoneae & Ruscaceae & 1 & 1 & & \\
\hline Dicotyledoneae & Rutaceae & 13 & 29 & 3 & 10,3 \\
\hline Dicotyledoneae & Sabiaceae & 1 & 1 & & \\
\hline Pteridophyta & Saccolomataceae & 1 & 3 & & \\
\hline Dicotyledoneae & Salicaceae & 8 & 44 & 14 & 31,8 \\
\hline Pteridophyta & Salviniaceae & 1 & 4 & & \\
\hline Dicotyledoneae & Samolaceae & 1 & 5 & 3 & 60,0 \\
\hline Dicotyledoneae & Santalaceae & 1 & 1 & 1 & 100,0 \\
\hline Dicotyledoneae & Sapindaceae & 23 & 97 & 12 & 12,4 \\
\hline Dicotyledoneae & Sapotaceae & 7 & 26 & 2 & 7,7 \\
\hline Dicotyledoneae & Saxifragaceae & 5 & 7 & 4 & 57,1 \\
\hline Pteridophyta & Schizaeaceae & 2 & 4 & & \\
\hline Dicotyledoneae & Schlegeliaceae & 1 & 1 & 1 & 100,0 \\
\hline Dicotyledoneae & Schoepfiaceae & 3 & 21 & 18 & 85,7 \\
\hline Dicotyledoneae & Scrophulariaceae & 5 & 30 & 13 & 43,3 \\
\hline Pteridophyta & Selaginellaceae & 1 & 18 & 2 & 11,1 \\
\hline Dicotyledoneae & Simaroubaceae & 6 & 13 & 3 & 23,1 \\
\hline Dicotyledoneae & Siparunaceae & 1 & 1 & & \\
\hline Monocotyledoneae & Smilacaceae & 1 & 14 & 2 & 14,3 \\
\hline Dicotyledoneae & Solanaceae & 40 & 496 & 237 & 47,8 \\
\hline Dicotyledoneae & Sphenocleaceae & 1 & 1 & & \\
\hline Dicotyledoneae & Sterculiaceae & 7 & 72 & 16 & 22,2 \\
\hline Dicotyledoneae & Stylidiaceae & 1 & 1 & 1 & 100,0 \\
\hline Dicotyledoneae & Styracaceae & 1 & 9 & & \\
\hline Dicotyledoneae & Symplocaceae & 1 & 19 & 3 & 15,8 \\
\hline Dicotyledoneae & Talinaceae & 1 & 5 & 1 & 20,0 \\
\hline Dicotyledoneae & Tamaricaceae & 1 & 1 & & \\
\hline Monocotyledoneae & Tecophilaeaceae & 3 & 9 & 8 & 88,9 \\
\hline Pteridophyta & Tectariaceae & 1 & 3 & & \\
\hline Dicotyledoneae & Tetrachondraceae & 1 & 1 & 1 & 100,0 \\
\hline Dicotyledoneae & Theaceae & 2 & 2 & & \\
\hline Pteridophyta & Thelypteridaceae & 2 & 60 & 9 & 15,0 \\
\hline Dicotyledoneae & Theophrastaceae & 1 & 1 & & \\
\hline Dicotyledoneae & Thesiaceae & 1 & 1 & & \\
\hline Dicotyledoneae & Thymelaeaceae & 3 & 8 & 3 & 37,5 \\
\hline Dicotyledoneae & Tiliaceae & 4 & 15 & 1 & 6,7 \\
\hline Dicotyledoneae & Tribelaceae & 1 & 1 & 1 & 100,0 \\
\hline Dicotyledoneae & Trigoniaceae & 1 & 2 & & \\
\hline Monocotyledoneae & Triuridaceae & 2 & 2 & & \\
\hline Dicotyledoneae & Tropaeolaceae & 1 & 33 & 28 & 84,8 \\
\hline Dicotyledoneae & Turneraceae & 2 & 31 & 6 & 19,4 \\
\hline Monocotyledoneae & Typhaceae & 1 & 4 & & \\
\hline
\end{tabular}




\begin{tabular}{llcccc}
\hline Group & Family & Genera & Species & Endemics & \% Endemism \\
\hline Dicotyledoneae & Ulmaceae & 1 & 1 & & \\
Dicotyledoneae & Urticaceae & 8 & 40 & 11 & 27,5 \\
Dicotyledoneae & Valerianaceae & 4 & 88 & 67 & 76,1 \\
Monocotyledoneae & Velloziaceae & 2 & 3 & 2 & 66,7 \\
Dicotyledoneae & Verbenaceae & 26 & 244 & 130 & 53,3 \\
Dicotyledoneae & Violaceae & 5 & 131 & 107 & 81,7 \\
Dicotyledoneae & Viscaceae & 1 & 34 & 1 & 2,9 \\
Dicotyledoneae & Vitaceae & 3 & 16 & & \\
Pteridophyta & Vittariaceae & 5 & 8 & & 100,0 \\
Dicotyledoneae & Vivianiaceae & 4 & 7 & 7 & 16,7 \\
Dicotyledoneae & Vochysiaceae & 3 & 12 & 2 & 80,0 \\
Dicotyledoneae & Winteraceae & 1 & 5 & 4 & \\
Pteridophyta & Woodsiaceae & 2 & 3 & & 16,7 \\
Dicotyledoneae & Ximeniaceae & 1 & 2 & & \\
Monocotyledoneae & Xyridaceae & 1 & 24 & 4 & 500,0 \\
Monocotyledoneae & Zannichelliaceae & 1 & 1 & & \\
Monocotyledoneae & Zingiberaceae & 1 & 1 & & \\
Monocotyledoneae & Zosteraceae & 1 & 1 & 1 & \\
Dicotyledoneae & Zygophyllaceae & 9 & 20 & 10 & \\
\hline Total & & 2678 & 18138 & 7798 & \\
\hline
\end{tabular}

Table 4 - Argentina, number of families, genera, species, and endemic species.

\begin{tabular}{llcccc}
\hline Group & Family & Genera & Species & Endemics & \%Endemics \\
\hline Dicotyledoneae & Acanthaceae & 16 & 77 & 6 & 7,8 \\
Dicotyledoneae & Aceraceae & 1 & 2 & & \\
Dicotyledoneae & Achatocarpaceae & 1 & 2 & & \\
Dicotyledoneae & Adoxaceae & 2 & 3 & & \\
Dicotyledoneae & Aextoxicaceae & 1 & 1 & & \\
Monocotyledoneae & Agapanthaceae & 1 & 1 & & \\
Monocotyledoneae & Agavaceae & 2 & 3 & & 10,0 \\
Dicotyledoneae & Aizoaceae & 7 & 10 & 1 & \\
Monocotyledoneae & Alismataceae & 4 & 15 & & 7,7 \\
Monocotyledoneae & Alstroemeriaceae & 2 & 13 & 1 & 45,3 \\
Dicotyledoneae & Amaranthaceae & 13 & 98 & 15 & 100,0 \\
Monocotyledoneae & Amaryllidaceae & 19 & 121 & 55 & 21,4 \\
Dicotyledoneae & Anacampserotaceae & 2 & 3 & 3 & \\
Dicotyledoneae & Anacardiaceae & 6 & 28 & 6 & 50,0 \\
Pteridophyta & Anemiaceae & 1 & 7 & & 16,7 \\
Dicotyledoneae & Annonaceae & 2 & 5 & & \\
Monocotyledoneae & Anthericaceae & 1 & 6 & 3 & \\
Dicotyledoneae & Apiaceae & 42 & 150 & 25 & \\
\hline
\end{tabular}




\begin{tabular}{|c|c|c|c|c|c|}
\hline Group & Family & Genera & Species & Endemics & \%Endemics \\
\hline Dicotyledoneae & Apocynaceae & 47 & 198 & 44 & 22,2 \\
\hline Dicotyledoneae & Aquifoliaceae & 1 & 6 & & \\
\hline Monocotyledoneae & Araceae & 13 & 17 & & \\
\hline Dicotyledoneae & Araliaceae & 6 & 8 & & \\
\hline Gymnospermae & Araucariaceae & 1 & 2 & & \\
\hline Monocotyledoneae & Arecaceae & 10 & 16 & 1 & 6,3 \\
\hline Dicotyledoneae & Aristolochiaceae & 1 & 21 & 1 & 4,8 \\
\hline Monocotyledoneae & Asparagaceae & 1 & 5 & & \\
\hline Monocotyledoneae & Asphodelaceae & 1 & 1 & & \\
\hline Pteridophyta & Aspleniaceae & 2 & 41 & 1 & 2,4 \\
\hline Monocotyledoneae & Asteliaceae & 1 & 1 & & \\
\hline Dicotyledoneae & Asteraceae & 282 & 1502 & 372 & 24,8 \\
\hline Pteridophyta & Athyriaceae & 3 & 8 & & \\
\hline Pteridophyta & Azollaceae & 1 & 2 & & \\
\hline Dicotyledoneae & Balanophoraceae & 3 & 4 & & \\
\hline Dicotyledoneae & Balsaminaceae & 1 & 2 & & \\
\hline Dicotyledoneae & Basellaceae & 2 & 4 & & \\
\hline Dicotyledoneae & Begoniaceae & 1 & 17 & 4 & 23,5 \\
\hline Dicotyledoneae & Berberidaceae & 1 & 20 & 8 & 40,0 \\
\hline Dicotyledoneae & Betulaceae & 2 & 3 & & \\
\hline Dicotyledoneae & Bignoniaceae & 22 & 57 & 1 & 1,8 \\
\hline Dicotyledoneae & Bixaceae & 1 & 1 & & \\
\hline Pteridophyta & Blechnaceae & 1 & 22 & & \\
\hline Dicotyledoneae & Bombacaceae & 3 & 5 & & \\
\hline Dicotyledoneae & Boraginaceae & 25 & 107 & 14 & 13,1 \\
\hline Dicotyledoneae & Brassicaceae & 63 & 229 & 61 & 26,6 \\
\hline Monocotyledoneae & Bromeliaceae & 13 & 111 & 39 & 35,1 \\
\hline Monocotyledoneae & Burmanniaceae & 3 & 5 & & \\
\hline Dicotyledoneae & Cabombaceae & 1 & 1 & & \\
\hline Dicotyledoneae & Cactaceae & 41 & 240 & 129 & 53,8 \\
\hline Dicotyledoneae & Calceolariaceae & 1 & 41 & 10 & 24,4 \\
\hline Dicotyledoneae & Callitrichaceae & 1 & 8 & & \\
\hline Dicotyledoneae & Calyceraceae & 6 & 35 & 12 & 34,3 \\
\hline Dicotyledoneae & Campanulaceae & 8 & 26 & 2 & 7,7 \\
\hline Dicotyledoneae & Cannabaceae & 1 & 2 & & \\
\hline Monocotyledoneae & Cannaceae & 1 & 4 & 1 & 25,0 \\
\hline Dicotyledoneae & Capparaceae & 10 & 19 & & \\
\hline Dicotyledoneae & Caprifoliaceae & 2 & 2 & & \\
\hline Dicotyledoneae & Cardiopteridaceae & 1 & 3 & & \\
\hline Dicotyledoneae & Caricaceae & 2 & 5 & & \\
\hline Dicotyledoneae & Caryophyllaceae & 25 & 122 & 22 & 18,0 \\
\hline Dicotyledoneae & Cecropiaceae & 1 & 1 & & \\
\hline Dicotyledoneae & Celastraceae & 7 & 16 & 3 & 18,8 \\
\hline
\end{tabular}




\begin{tabular}{|c|c|c|c|c|c|}
\hline Group & Family & Genera & Species & Endemics & \%Endemics \\
\hline Dicotyledoneae & Celtidaceae & 2 & 5 & & \\
\hline Monocotyledoneae & Centrolepidaceae & 1 & 1 & & \\
\hline Dicotyledoneae & Ceratophyllaceae & 1 & 2 & & \\
\hline Dicotyledoneae & Cervantesiaceae & 2 & 3 & & \\
\hline Dicotyledoneae & Chenopodiaceae & 16 & 93 & 29 & 31,2 \\
\hline Dicotyledoneae & Cistaceae & 1 & 1 & & \\
\hline Dicotyledoneae & Clethraceae & 1 & 1 & & \\
\hline Dicotyledoneae & Clusiaceae & 2 & 2 & & \\
\hline Dicotyledoneae & Cochlospermaceae & 1 & 1 & & \\
\hline Dicotyledoneae & Combretaceae & 2 & 6 & & \\
\hline Monocotyledoneae & Commelinaceae & 8 & 25 & & \\
\hline Dicotyledoneae & Convolvulaceae & 12 & 128 & 20 & 15,6 \\
\hline Dicotyledoneae & Coriariaceae & 1 & 1 & & \\
\hline Monocotyledoneae & Corsiaceae & 1 & 1 & & \\
\hline Monocotyledoneae & Costaceae & 1 & 1 & & \\
\hline Dicotyledoneae & Crassulaceae & 4 & 13 & 3 & 23,1 \\
\hline Dicotyledoneae & Cucurbitaceae & 21 & 51 & 4 & 7,8 \\
\hline Dicotyledoneae & Cunoniaceae & 3 & 4 & & \\
\hline Gymnospermae & Cupressaceae & 4 & 4 & & \\
\hline Pteridophyta & Cyatheaceae & 2 & 4 & & \\
\hline Monocotyledoneae & Cyperaceae & 24 & 314 & 17 & 5,4 \\
\hline Pteridophyta & Dennstaedtiaceae & 3 & 7 & & \\
\hline Dicotyledoneae & Desfontainiaceae & 1 & 1 & & \\
\hline Pteridophyta & Dicksoniaceae & 2 & 2 & & \\
\hline Pteridophyta & Didymochlaenaceae & 1 & 1 & & \\
\hline Dicotyledoneae & Dilleniaceae & 1 & 1 & & \\
\hline Monocotyledoneae & Dioscoreaceae & 1 & 34 & 7 & 20,6 \\
\hline Dicotyledoneae & Dipsacaceae & 3 & 4 & & \\
\hline Dicotyledoneae & Donatiaceae & 1 & 1 & & \\
\hline Dicotyledoneae & Droseraceae & 1 & 3 & & \\
\hline Pteridophyta & Dryopteridaceae & 10 & 34 & 1 & 2,9 \\
\hline Dicotyledoneae & Ebenaceae & 1 & 1 & & \\
\hline Dicotyledoneae & Elaeagnaceae & 1 & 1 & & \\
\hline Dicotyledoneae & Elaeocarpaceae & 4 & 4 & & \\
\hline Dicotyledoneae & Elatinaceae & 1 & 2 & 1 & 50,0 \\
\hline Dicotyledoneae & Empetraceae & 1 & 1 & & \\
\hline Dicotyledoneae & Epacridaceae & 1 & 1 & & \\
\hline Gymnospermae & Ephedraceae & 1 & 10 & 1 & 10,0 \\
\hline Pteridophyta & Equisetaceae & 1 & 2 & & \\
\hline Dicotyledoneae & Eremolepidaceae & 1 & 1 & & \\
\hline Dicotyledoneae & Ericaceae & 5 & 16 & & \\
\hline Monocotyledoneae & Eriocaulaceae & 2 & 8 & 1 & 12,5 \\
\hline Dicotyledoneae & Erythroxylaceae & 1 & 5 & & \\
\hline
\end{tabular}




\begin{tabular}{|c|c|c|c|c|c|}
\hline Group & Family & Genera & Species & Endemics & $\%$ Endemics \\
\hline Dicotyledoneae & Escalloniaceae & 1 & 16 & 1 & 6,3 \\
\hline Dicotyledoneae & Eucryphiaceae & 1 & 1 & & \\
\hline Dicotyledoneae & Euphorbiaceae & 29 & 218 & 30 & 13,8 \\
\hline Dicotyledoneae & Fabaceae & 119 & 760 & 160 & 21,1 \\
\hline Dicotyledoneae & Frankeniaceae & 1 & 7 & 3 & 42,9 \\
\hline Dicotyledoneae & Fumariaceae & 1 & 6 & & \\
\hline Dicotyledoneae & Gentianaceae & 11 & 44 & 15 & 34,1 \\
\hline Dicotyledoneae & Geraniaceae & 2 & 23 & 4 & 17,4 \\
\hline Dicotyledoneae & Gesneriaceae & 5 & 11 & & \\
\hline Pteridophyta & Gleicheniaceae & 2 & 3 & & \\
\hline Dicotyledoneae & Griseliniaceae & 1 & 2 & & \\
\hline Dicotyledoneae & Grossulariaceae & 1 & 6 & & \\
\hline Dicotyledoneae & Gunneraceae & 1 & 6 & & \\
\hline Dicotyledoneae & Halophytaceae & 1 & 1 & 1 & 100,0 \\
\hline Dicotyledoneae & Haloragaceae & 1 & 2 & & \\
\hline Monocotyledoneae & Heliconiaceae & 1 & 3 & & \\
\hline Monocotyledoneae & Herreriaceae & 2 & 4 & & \\
\hline Dicotyledoneae & Hippuridaceae & 1 & 1 & & \\
\hline Monocotyledoneae & Hyacinthaceae & 1 & 3 & & \\
\hline Dicotyledoneae & Hydnoraceae & 1 & 2 & & \\
\hline Dicotyledoneae & Hydrangeaceae & 1 & 1 & & \\
\hline Monocotyledoneae & Hydrocharitaceae & 5 & 6 & & \\
\hline Dicotyledoneae & Hydroleaceae & 1 & 2 & & \\
\hline Pteridophyta & Hymenophyllaceae & 5 & 29 & 1 & 3,4 \\
\hline Dicotyledoneae & Hypericaceae & 1 & 14 & & \\
\hline Monocotyledoneae & Hypoxidaceae & 2 & 4 & 1 & 25,0 \\
\hline Monocotyledoneae & Iridaceae & 23 & 121 & 33 & 27,3 \\
\hline Pteridophyta & Isoëtaceae & 1 & 7 & 4 & 57,1 \\
\hline Dicotyledoneae & Juglandaceae & 2 & 2 & & \\
\hline Monocotyledoneae & Juncaceae & 7 & 52 & 5 & 9,6 \\
\hline Monocotyledoneae & Juncaginaceae & 2 & 5 & & \\
\hline Dicotyledoneae & Krameriaceae & 1 & 1 & & \\
\hline Dicotyledoneae & Lacistemataceae & 1 & 2 & & \\
\hline Dicotyledoneae & Lamiaceae & 31 & 112 & 3 & 2,7 \\
\hline Dicotyledoneae & Lardizabalaceae & 1 & 1 & & \\
\hline Dicotyledoneae & Lauraceae & 5 & 14 & & \\
\hline Monocotyledoneae & Laxmanniaceae & 1 & 1 & 1 & 100,0 \\
\hline Dicotyledoneae & Ledocarpaceae & 1 & 6 & 3 & 50,0 \\
\hline Monocotyledoneae & Lemnaceae & 4 & 10 & & \\
\hline Dicotyledoneae & Lentibulariaceae & 2 & 16 & & \\
\hline Monocotyledoneae & Limnocharitaceae & 2 & 5 & & \\
\hline Dicotyledoneae & Linaceae & 2 & 10 & & \\
\hline
\end{tabular}




\begin{tabular}{|c|c|c|c|c|c|}
\hline Group & Family & Genera & Species & Endemics & \%Endemics \\
\hline Dicotyledoneae & Linderniaceae & 1 & 1 & & \\
\hline Pteridophyta & Lindsaeaceae & 1 & 2 & & \\
\hline Dicotyledoneae & Loasaceae & 4 & 37 & 6 & 16,2 \\
\hline Dicotyledoneae & Loganiaceae & 2 & 10 & & \\
\hline Dicotyledoneae & Loranthaceae & 5 & 8 & & \\
\hline Monocotyledoneae & Luzuriagaceae & 1 & 2 & & \\
\hline Pteridophyta & Lycopodiaceae & 9 & 22 & 1 & 4,5 \\
\hline Pteridophyta & Lygodiaceae & 1 & 1 & 2 & 200,0 \\
\hline Dicotyledoneae & Lythraceae & 8 & 35 & & \\
\hline Dicotyledoneae & Malesherbiaceae & 1 & 3 & & \\
\hline Dicotyledoneae & Malpighiaceae & 23 & 46 & 3 & 6,5 \\
\hline Dicotyledoneae & Malvaceae & 34 & 233 & 53 & 22,7 \\
\hline Monocotyledoneae & Marantaceae & 4 & 6 & & \\
\hline Pteridophyta & Marattiaceae & 1 & 1 & & \\
\hline Pteridophyta & Marsileaceae & 3 & 5 & & \\
\hline Dicotyledoneae & Martyniaceae & 2 & 4 & 2 & 50,0 \\
\hline Monocotyledoneae & Mayacaceae & 1 & 2 & & \\
\hline Dicotyledoneae & Melastomataceae & 9 & 32 & 1 & 3,1 \\
\hline Dicotyledoneae & Meliaceae & 5 & 14 & 1 & 7,1 \\
\hline Dicotyledoneae & Menispermaceae & 3 & 6 & & \\
\hline Dicotyledoneae & Menyanthaceae & 1 & 1 & & \\
\hline Dicotyledoneae & Microteaceae & 1 & 1 & & \\
\hline Dicotyledoneae & Misodendraceae & 1 & 7 & & \\
\hline Dicotyledoneae & Molluginaceae & 2 & 3 & & \\
\hline Dicotyledoneae & Monimiaceae & 2 & 2 & & \\
\hline Dicotyledoneae & Montiaceae & 8 & 36 & 1 & 2,8 \\
\hline Dicotyledoneae & Moraceae & 6 & 14 & & \\
\hline Dicotyledoneae & Muntingiaceae & 1 & 1 & & \\
\hline Dicotyledoneae & Myoporaceae & 1 & 1 & & \\
\hline Dicotyledoneae & Myricaceae & 1 & 1 & & \\
\hline Dicotyledoneae & Myrsinaceae & 1 & 6 & & \\
\hline Dicotyledoneae & Myrtaceae & 23 & 65 & 2 & 3,1 \\
\hline Monocotyledoneae & Najadaceae & 1 & 2 & & \\
\hline Dicotyledoneae & Nanodeaceae & 1 & 1 & & \\
\hline Pteridophyta & Nephrolepidaceae & 1 & 1 & & \\
\hline Dicotyledoneae & Nothofagaceae & 2 & 6 & & \\
\hline Dicotyledoneae & Nyctaginaceae & 8 & 19 & 2 & 10,5 \\
\hline Dicotyledoneae & Nymphaeaceae & 2 & 6 & & \\
\hline Dicotyledoneae & Ochnaceae & 1 & 2 & & \\
\hline Dicotyledoneae & Oleaceae & 5 & 13 & 1 & 7,7 \\
\hline Dicotyledoneae & Onagraceae & 7 & 70 & 9 & 12,9 \\
\hline Pteridophyta & Ophioglossaceae & 2 & 9 & & \\
\hline Dicotyledoneae & Opiliaceae & 1 & 1 & & \\
\hline
\end{tabular}




\begin{tabular}{|c|c|c|c|c|c|}
\hline Group & Family & Genera & Species & Endemics & \%Endemics \\
\hline Monocotyledoneae & Orchidaceae & 93 & 281 & 18 & 6,4 \\
\hline Dicotyledoneae & Orobanchaceae & 9 & 28 & 1 & 3,6 \\
\hline Pteridophyta & Osmundaceae & 2 & 2 & & \\
\hline Dicotyledoneae & Oxalidaceae & 2 & 73 & 10 & 13,7 \\
\hline Dicotyledoneae & Papaveraceae & 6 & 12 & & \\
\hline Pteridophyta & Parkeriaceae & 1 & 1 & & \\
\hline Dicotyledoneae & Parnassiaceae & 1 & 1 & & \\
\hline Dicotyledoneae & Passifloraceae & 1 & 20 & & \\
\hline Dicotyledoneae & Pentaphylacaceae & 1 & 1 & & \\
\hline Monocotyledoneae & Philesiaceae & 1 & 1 & & \\
\hline Dicotyledoneae & Phrymaceae & 2 & 6 & & \\
\hline Dicotyledoneae & Phyllanthaceae & 2 & 10 & & \\
\hline Dicotyledoneae & Phytolaccaceae & 6 & 13 & 1 & 7,7 \\
\hline Dicotyledoneae & Picrodendraceae & 1 & 1 & & \\
\hline Gymnospermae & Pinaceae & 2 & 4 & & \\
\hline Dicotyledoneae & Piperaceae & 2 & 42 & 5 & 11,9 \\
\hline Dicotyledoneae & Plantaginaceae & 26 & 116 & 13 & 11,2 \\
\hline Dicotyledoneae & Plumbaginaceae & 3 & 4 & & \\
\hline Monocotyledoneae & Poaceae & 187 & 1170 & 165 & 14,1 \\
\hline Gymnospermae & Podocarpaceae & 4 & 6 & & \\
\hline Dicotyledoneae & Podostemaceae & 6 & 9 & 1 & 11,1 \\
\hline Dicotyledoneae & Polemoniaceae & 7 & 11 & 2 & 18,2 \\
\hline Dicotyledoneae & Polygalaceae & 6 & 61 & 21 & 34,4 \\
\hline Dicotyledoneae & Polygonaceae & 12 & 58 & 2 & 3,4 \\
\hline Pteridophyta & Polypodiaceae & 12 & 44 & 1 & 2,3 \\
\hline Monocotyledoneae & Pontederiaceae & 3 & 10 & & \\
\hline Dicotyledoneae & Portulacaceae & 1 & 24 & 8 & 33,3 \\
\hline Monocotyledoneae & Potamogetonaceae & 2 & 12 & & \\
\hline Dicotyledoneae & Primulaceae & 6 & 11 & & \\
\hline Dicotyledoneae & Proteaceae & 6 & 8 & & \\
\hline Pteridophyta & Psilotaceae & 1 & 1 & & \\
\hline Pteridophyta & Pteridaceae & 17 & 75 & 3 & 4,0 \\
\hline Dicotyledoneae & Quillajaceae & 1 & 1 & & \\
\hline Dicotyledoneae & Rafflesiaceae & 1 & 2 & & \\
\hline Dicotyledoneae & Ranunculaceae & 12 & 55 & 3 & 5,5 \\
\hline Dicotyledoneae & Resedaceae & 1 & 1 & & \\
\hline Dicotyledoneae & Rhamnaceae & 14 & 26 & 4 & 15,4 \\
\hline Dicotyledoneae & Rosaceae & 22 & 95 & 10 & 10,5 \\
\hline Dicotyledoneae & Rubiaceae & 41 & 133 & 9 & 6,8 \\
\hline Monocotyledoneae & Ruppiaceae & 1 & 3 & & \\
\hline Monocotyledoneae & Ruscaceae & 1 & 1 & & \\
\hline Dicotyledoneae & Rutaceae & 8 & 17 & & \\
\hline Pteridophyta & Saccolomataceae & 1 & 1 & & \\
\hline
\end{tabular}




\begin{tabular}{|c|c|c|c|c|c|}
\hline Group & Family & Genera & Species & Endemics & \%Endemics \\
\hline Dicotyledoneae & Salicaceae & 8 & 30 & 1 & 3,3 \\
\hline Pteridophyta & Salviniaceae & 1 & 4 & & \\
\hline Dicotyledoneae & Samolaceae & 1 & 3 & & \\
\hline Dicotyledoneae & Santalaceae & 1 & 1 & & \\
\hline Dicotyledoneae & Sapindaceae & 17 & 46 & 2 & 4,3 \\
\hline Dicotyledoneae & Sapotaceae & 3 & 7 & & \\
\hline Dicotyledoneae & Saxifragaceae & 5 & 6 & & \\
\hline Pteridophyta & Schizaeaceae & 1 & 1 & & \\
\hline Dicotyledoneae & Schoepfiaceae & 3 & 6 & 1 & 16,7 \\
\hline Dicotyledoneae & Scrophulariaceae & 3 & 17 & 1 & 5,9 \\
\hline Pteridophyta & Selaginellaceae & 1 & 8 & & \\
\hline Dicotyledoneae & Simaroubaceae & 6 & 8 & & \\
\hline Monocotyledoneae & Smilacaceae & 1 & 5 & & \\
\hline Dicotyledoneae & Solanaceae & 35 & 322 & 87 & 27,0 \\
\hline Dicotyledoneae & Sphenocleaceae & 1 & 1 & & \\
\hline Dicotyledoneae & Sterculiaceae & 6 & 45 & 3 & 6,7 \\
\hline Dicotyledoneae & Stylidiaceae & 1 & 1 & & \\
\hline Dicotyledoneae & Styracaceae & 1 & 2 & & \\
\hline Dicotyledoneae & Symplocaceae & 1 & 2 & & \\
\hline Dicotyledoneae & Talinaceae & 1 & 3 & & \\
\hline Dicotyledoneae & Tamaricaceae & 1 & 1 & & \\
\hline Pteridophyta & Tectariaceae & 1 & 1 & & \\
\hline Dicotyledoneae & Tetrachondraceae & 1 & 1 & & \\
\hline Dicotyledoneae & Theaceae & 1 & 1 & & \\
\hline Pteridophyta & Thelypteridaceae & 2 & 36 & 2 & 5,6 \\
\hline Dicotyledoneae & Thymelaeaceae & 3 & 3 & & \\
\hline Dicotyledoneae & Tiliaceae & 4 & 12 & & \\
\hline Dicotyledoneae & Tribelaceae & 1 & 1 & & \\
\hline Dicotyledoneae & Trigoniaceae & 1 & 1 & & \\
\hline Monocotyledoneae & Triuridaceae & 1 & 1 & & \\
\hline Dicotyledoneae & Tropaeolaceae & 1 & 16 & 7 & 43,8 \\
\hline Dicotyledoneae & Turneraceae & 2 & 14 & & \\
\hline Monocotyledoneae & Typhaceae & 1 & 4 & & \\
\hline Dicotyledoneae & Ulmaceae & 1 & 1 & & \\
\hline Dicotyledoneae & Urticaceae & 7 & 25 & 2 & 8,0 \\
\hline Dicotyledoneae & Valerianaceae & 4 & 52 & 16 & 30,8 \\
\hline Monocotyledoneae & Velloziaceae & 1 & 2 & 1 & 50,0 \\
\hline Dicotyledoneae & Verbenaceae & 23 & 169 & 42 & 24,9 \\
\hline Dicotyledoneae & Violaceae & 4 & 70 & 29 & 41,4 \\
\hline Dicotyledoneae & Viscaceae & 1 & 16 & & \\
\hline Dicotyledoneae & Vitaceae & 3 & 10 & & \\
\hline Pteridophyta & Vittariaceae & 2 & 3 & & \\
\hline Dicotyledoneae & Vivianiaceae & 2 & 3 & & \\
\hline
\end{tabular}




\begin{tabular}{llcccc}
\hline Group & Family & Genera & Species & Endemics & \%Endemics \\
\hline Dicotyledoneae & Vochysiaceae & 1 & 1 & & \\
Dicotyledoneae & Winteraceae & 1 & 1 & & \\
Pteridophyta & Woodsiaceae & 2 & 3 & & \\
Dicotyledoneae & Ximeniaceae & 1 & 1 & & \\
Monocotyledoneae & Xyridaceae & 1 & 7 & & \\
Monocotyledoneae & Zannichelliaceae & 1 & 1 & & \\
Monocotyledoneae & Zingiberaceae & 1 & 1 & & 33,3 \\
Dicotyledoneae & Zygophyllaceae & 6 & 15 & 5 & \\
\hline Total & & 2090 & 10006 & 1749 & \\
\hline
\end{tabular}

Halophytaceae and the endemic genera are 44 ( $2.10 \%$ of the total of Argentinian genera), with the Asteraceae leading in number of endemic genera (12), followed by Brassicaceae (8), Cactaceae (4), Fabaceae and Verbenaceae (3) (Tab. 5); the total endemic species for Argentina is 1,749 (17,48\% of the total number of species for this country).

Endemism in the Biogeographic Regions Of the eight families endemic from the Southern Cone, the monotypic Lactoridaceae is exclusive from the Robison Crusoe Island (Más a Tierra), in the Juan Fernández Archipelago (Chile), included in the Temperate-humid region of the Pacific, with a flora related to the Subantarctic phytogeographic province, however counting with subtropical and other specific elements from the Pacific Islands. The Francoaceae, with two monotypic genera and the monotypic Gomortegaceae are exclusive from the mediterranean region of Central Chile. All four endemic families shared between Argentina and Chile are from the Subantartic forests, the Aextoxicaceae and Tribelaceae are monotypic and the Philesiaceae comprises two monotypic genera, while the Misodendraceae has a single genus and eight hemiparasitic species. The Halophytaceae, the only endemic family from Argentina, is exclusive from the Monte region, in arid pre-montane areas.

In the South-Central dry Andes, which include the Puna, Prepuna and high-andean regions in northern Argentina and Chile, there are several endemic genera of Asteraceae such as Cabreraea Bonifacino, Dolichlasium Lag. and Famatinanthus Ariza \& S.E. Freire, of Brassicaceae, as Lithodraba Boelcke, Parodiodoxa O.E. Schulz, Sarcodraba Gilg \& Muschl. and Zuloagocardamum
Salariato \& Al-Shehbaz and of Montiaceae, as Schreiteria Carolin, amongst others. Genera that are characteristic but not endemic to the Southern Cone are Clinanthus Herb. and Hieronymiella Pax (Amaryllidaceae), Chiliotrichiopsis Cabrera, Cuatrecasasiella H. Rob., Urmenetea Phil. and Werneria Kunth (Asteraceae), Aschersoniodoxa Gilg $\&$ Muschl., Mancoa Wedd. and Polypsecadium O. E. Schulz (Brassicaceae), Pycnophyllum J. Rémy (Caryophyllaceae), Cardenanthus R.C. Forster and Mastigostyla I.M. Johnst. (Iridaceae), Nototriche Turcz. and Tarasa Phil. (Malvaceae), Hypseocharis J. Rémy (Oxalidaceae), Aa Juss. and Myrosmodes Rchb. f. (Orchidaceae), Lenzia Phil. (Montiaceae) and Anthochloa Nees \& Meyen and Aciachne Benth. (Poaceae), among others. Some genera are common between the high Andes and the Patagonic region, such as Adesmia (Fabaceae), Azorella Lam. and Mulinum Pers. (Apiaceae), Chuquiraga Juss. and Nardophyllum Hook. \& Arn. (Asteraceae), Fabiana Ruiz \& Pav. (Solanaceae) and Junellia Moldenke (Verbenaceae). Among the genera that are endemic to the central Andes of Mendoza and San Juan (Argentina) are Haroldia Bonifacino, Huarpea Cabrera and Katinasia Bonifacino (Asteraceae).

The North-Central Humid Andes or Yungas show few endemic genera, one of the examples being Austropeucedanum Mathias \& Constance (Apiaceae), and this may be explained by the continuation of the Yungas into bordering areas of Bolivia. The characteristic or exclusive elements within this region include holartic families such as BetulaWceae (Alnus Mill.) and Juglandaceae (Juglans L.) and other families that reach northern Argentina, such as Myricaceae (Myrica L.), Pentaphylacaceae (Ternstroemia Mutis ex L. f.) and Velloziaceae (Barbaceniopsis L.B. Sm.). 
Table 5 - Southern Cone, endemic genera.

\begin{tabular}{|c|c|c|c|c|c|c|}
\hline Family & Genus & No'Spp. & & & untries & \\
\hline Aextoxicaceae & Aextoxicon & 1 & Argentina & & Chile & \\
\hline Alstroemeriaceae & Leontochir & 1 & & & Chile & \\
\hline Amaranthaceae & Quaternella & 1 & & S Brasil & & \\
\hline \multirow[t]{16}{*}{ Amaryllidaceae } & Ancrumia & 1 & & & Chile & \\
\hline & Beauverdia & 4 & Argentina & S Brasil & & Uruguay \\
\hline & Eithea & 1 & & S Brasil & & \\
\hline & Famatina & 2 & Argentina & & Chile & \\
\hline & Gethyum & 2 & & & Chile & \\
\hline & Gilliesia & 5 & Argentina & & Chile & \\
\hline & Ipheion & 4 & Argentina & & Chile & Uruguay \\
\hline & Leucocoryne & 45 & & & Chile & \\
\hline & Miersia & 4 & & & Chile & \\
\hline & Phycella & 6 & Argentina & & Chile & \\
\hline & Placea & 5 & & & Chile & \\
\hline & Solaria & 3 & Argentina & & Chile & \\
\hline & Speea & 1 & & & Chile & \\
\hline & Traubia & 1 & & & Chile & \\
\hline & Tristagma & 20 & Argentina & & Chile & \\
\hline & Zoellnerallium & 2 & Argentina & & Chile & \\
\hline \multirow[t]{11}{*}{ Apiaceae } & Asteriscium & 9 & Argentina & & Chile & \\
\hline & Austropeucedanum & 1 & Argentina & & & \\
\hline & Bolax & 2 & Argentina & & Chile & \\
\hline & Diposis & 3 & Argentina & & Chile & Uruguay \\
\hline & Gymnophyton & 6 & Argentina & & Chile & \\
\hline & Homalocarpus & 6 & & & Chile & \\
\hline & Huanaca & 4 & Argentina & & Chile & \\
\hline & Laretia & 1 & Argentina & & Chile & \\
\hline & Notiosciadium & 1 & Argentina & & & Uruguay \\
\hline & Oligocladus & 1 & Argentina & & & \\
\hline & Pozoa & 2 & Argentina & & Chile & \\
\hline \multirow[t]{3}{*}{ Apocynaceae } & Diplolepis & 14 & Argentina & & Chile & \\
\hline & Elytropus & 1 & & & Chile & \\
\hline & Rhyssostelma & 1 & & & & Uruguay \\
\hline Araceae & Mangonia & 2 & & S Brasil & & Uruguay \\
\hline \multirow[t]{2}{*}{ Arecaceae } & Juania & 1 & & & Chile & \\
\hline & Jubaea & 1 & & & Chile & \\
\hline \multirow[t]{5}{*}{ Asteraceae } & Acrisione & 1 & Argentina & & Chile & \\
\hline & Ameghinoa & 1 & Argentina & & & \\
\hline & Asteropsis & 1 & & S Brasil & & Uruguay \\
\hline & Aylacophora & 1 & Argentina & & & \\
\hline & Brachyclados & 3 & Argentina & & Chile & \\
\hline
\end{tabular}




\begin{tabular}{|c|c|c|c|c|c|c|}
\hline Family & Genus & $\mathrm{N}^{\circ}$ Spp. & & & Countries & \\
\hline & Burkartia & 1 & Argentina & & & \\
\hline & Cabreraea & 1 & Argentina & & & \\
\hline & Calopappus & 1 & & & Chile & \\
\hline & Centaurodendron & 2 & & & Chile & \\
\hline & Chiliophyllum & 3 & Argentina & & Chile & \\
\hline & Chiliotrichum & 2 & Argentina & & Chile & \\
\hline & Criscia & 1 & Argentina & S Brasil & & Uruguay \\
\hline & Dendroseris & 11 & & & Chile & \\
\hline & Dolichlasium & 1 & Argentina & & & \\
\hline & Doniophyton & 2 & Argentina & & Chile & \\
\hline & Duseniella & 1 & Argentina & & & \\
\hline & Eriachaenium & 1 & Argentina & & & \\
\hline & Famtinanthus & 1 & Argentina & & & \\
\hline & Gamochaetopsis & 1 & Argentina & & Chile & \\
\hline & Guynesomia & 1 & & & Chile & \\
\hline & Gypothamnium & 1 & & & Chile & \\
\hline & Haroldia & 1 & Argentina & & & \\
\hline & Heterothalamulopsis & 1 & & S Brasil & & \\
\hline & Huarpea & 1 & Argentina & & & \\
\hline & Ianthopappus & 1 & Argentina & S Brasil & & Uruguay \\
\hline & Katinasia & 1 & Argentina & & & \\
\hline & Kieslingia & 1 & & & Chile & \\
\hline & Lepidophyllum & 1 & Argentina & & Chile & \\
\hline & Leptinella & 1 & Argentina & & Chile & \\
\hline & Leptocarpha & 1 & & & Chile & \\
\hline & Leunisia & 1 & & & Chile & \\
\hline & Lycapsus & 1 & & & Chile & \\
\hline & Macrachaenium & 1 & Argentina & & & \\
\hline & Marticorenia & 1 & & & Chile & \\
\hline & Microgyne & 1 & Argentina & S Brasil & & Uruguay \\
\hline & Micropsis & 5 & Argentina & & Chile & Uruguay \\
\hline & Moscharia & 2 & & & Chile & \\
\hline & Oxyphyllum & 1 & & & Chile & \\
\hline & Panphalea & 9 & Argentina & S Brasil & & Uruguay \\
\hline & Pleocarphus & 1 & & & Chile & \\
\hline & Podanthus & 2 & & & Chile & \\
\hline & Sommerfeltia & 2 & Argentina & S Brasil & & Uruguay \\
\hline & Thamnoseris & 1 & & & Chile & \\
\hline & Triptilion & 7 & Argentina & & Chile & \\
\hline & Urmenetea & 1 & Argentina & & Chile & \\
\hline & Yunquea & 1 & & & Chile & \\
\hline Bignoniaceae & Campsidium & 1 & Argentina & & Chile & \\
\hline Boraginaceae & Ixorhea & 1 & Argentina & & & \\
\hline
\end{tabular}




\begin{tabular}{|c|c|c|c|c|}
\hline Family & Genus & No'Spp. & & Countries \\
\hline & Nesocaryum & 1 & & Chile \\
\hline & Selkirkia & 1 & & Chile \\
\hline \multirow[t]{13}{*}{ Brassicaceae } & Chilocardamum & 4 & Argentina & \\
\hline & Delpinophytum & 1 & Argentina & \\
\hline & Hollermayera & 1 & & Chile \\
\hline & Ivania & 1 & & Chile \\
\hline & Lithodraba & 1 & Argentina & \\
\hline & Onuris & 5 & Argentina & Chile \\
\hline & Parodiodoxa & 1 & Argentina & \\
\hline & Phlebolobium & 1 & Argentina & \\
\hline & Sarcodraba & 4 & Argentina & \\
\hline & Schizopetalon & 10 & Argentina & Chile \\
\hline & Trichotolinum & 1 & Argentina & \\
\hline & Xerodraba & 7 & Argentina & Chile \\
\hline & Zuloagocardamum & 1 & Argentina & \\
\hline \multirow[t]{2}{*}{ Bromeliaceae } & Fascicularia & 1 & & Chile \\
\hline & Ochagavia & 4 & & Chile \\
\hline \multirow[t]{10}{*}{ Cactaceae } & Acanthocalycium & 5 & Argentina & \\
\hline & Austrocactus & 4 & Argentina & Chile \\
\hline & Denmoza & 1 & Argentina & \\
\hline & Eriosyce & 5 & & Chile \\
\hline & Maihuenia & 2 & Argentina & Chile \\
\hline & Pterocactus & 9 & Argentina & Chile \\
\hline & Pyrrhocactus & 27 & Argentina & Chile \\
\hline & Setiechinopsis & 1 & Argentina & \\
\hline & Tephrocactus & 7 & Argentina & \\
\hline & Thelocephala & 6 & Argentina & Chile \\
\hline \multirow[t]{3}{*}{ Calyceraceae } & Boopis & 13 & Argentina & Chile \\
\hline & Gamocarpha & 6 & Argentina & Chile \\
\hline & Nastanthus & 9 & Argentina & Chile \\
\hline Campanulaceae & Cyphocarpus & 3 & & Chile \\
\hline \multirow[t]{2}{*}{ Caryophyllaceae } & Philippiella & 1 & Argentina & Chile \\
\hline & Reicheella & 1 & & Chile \\
\hline Corsiaceae & Arachnitis & 1 & Argentina & Chile \\
\hline Cucurbitaceae & Halosicyos & 1 & Argentina & \\
\hline \multirow[t]{3}{*}{ Cupressaceae } & Austrocedrus & 1 & Argentina & Chile \\
\hline & Fitzroya & 1 & Argentina & Chile \\
\hline & Pilgerodendron & 1 & Argentina & Chile \\
\hline Dicksoniaceae & Thyrsopteris & 1 & & Chile \\
\hline Epacridaceae & Lebetanthus & 1 & Argentina & Chile \\
\hline Escalloniaceae & Valdivia & 1 & & Chile \\
\hline \multirow[t]{2}{*}{ Euphorbiaceae } & Adenopeltis & 1 & & Chile \\
\hline & Avellanita & 1 & & Chile \\
\hline
\end{tabular}




\begin{tabular}{|c|c|c|c|c|c|c|c|}
\hline Family & Genus & No'Spp. & & & ountries & & \\
\hline & Colliguaja & 5 & Argentina & S Brasil & Chile & & \\
\hline \multirow[t]{6}{*}{ Fabaceae } & Anarthrophyllum & 15 & Argentina & & Chile & & \\
\hline & Balsamocarpon & 1 & Argentina & & Chile & & \\
\hline & Lophocarpinia & 1 & Argentina & & & Paraguay & \\
\hline & Ramorinoa & 1 & Argentina & & & & \\
\hline & Stenodrepanum & 1 & Argentina & & & & \\
\hline & Zuccagnia & 1 & Argentina & & & & \\
\hline \multirow[t]{2}{*}{ Francoaceae } & Francoa & 1 & & & Chile & & \\
\hline & Tetilla & 1 & & & Chile & & \\
\hline \multirow[t]{3}{*}{ Gesneriaceae } & Asteranthera & 1 & Argentina & & Chile & & \\
\hline & Mitraria & 1 & Argentina & & Chile & & \\
\hline & Sarmienta & 1 & & & Chile & & \\
\hline Gomortegaceae & Gomortega & 1 & & & Chile & & \\
\hline Halophytaceae & Halophytum & 1 & Argentina & & & & \\
\hline \multirow[t]{2}{*}{ Hymenophyllaceae } & Hymenoglossum & 1 & Argentina & & Chile & & \\
\hline & Serpyllopsis & 1 & Argentina & & Chile & & \\
\hline \multirow[t]{2}{*}{ Iridaceae } & Solenomelus & 2 & Argentina & & Chile & & \\
\hline & Tapeinia & 1 & Argentina & & & & \\
\hline Juncaginaceae & Tetroncium & 1 & Argentina & & Chile & & \\
\hline Lactoridaceae & Lactoris & 1 & & & Chile & & \\
\hline \multirow[t]{2}{*}{ Lamiaceae } & Cuminia & 1 & & & Chile & & \\
\hline & Kurzamra & 1 & Argentina & & Chile & & \\
\hline \multirow[t]{2}{*}{ Lardizabalaceae } & Boquila & 1 & Argentina & & Chile & & \\
\hline & Lardizabala & 1 & Argentina & & Chile & & \\
\hline Laxmanniaceae & Trichopetalum & 2 & Argentina & & Chile & & \\
\hline \multirow[t]{2}{*}{ Loasaceae } & Huidobria & 2 & & & Chile & & \\
\hline & Scyphanthus & 1 & & & Chile & & \\
\hline \multirow[t]{2}{*}{ Loranthaceae } & Desmaria & 1 & & & Chile & & \\
\hline & Notanthera & 1 & & & Chile & & \\
\hline \multirow[t]{6}{*}{ Malpighiaceae } & Cordobia & 1 & Argentina & & & Paraguay & \\
\hline & Dinemagonum & 1 & & & Chile & & \\
\hline & Dinemandra & 1 & & & Chile & & \\
\hline & Gallardoa & 1 & Argentina & & & & \\
\hline & Peregrina & 1 & Argentina & S Brasil & & Paraguay & \\
\hline & Tricomaria & 1 & Argentina & & & & \\
\hline \multirow[t]{7}{*}{ Malvaceae } & Bordasia & 1 & & & & Paraguay & \\
\hline & Calyculogygas & 1 & & S Brasil & & & Uruguay \\
\hline & Calyptraemalva & 1 & & S Brasil & & & \\
\hline & Corynabutilon & 7 & Argentina & & Chile & & \\
\hline & Lecanophora & 7 & Argentina & & Chile & & \\
\hline & Neobaclea & 1 & Argentina & & & & \\
\hline & Tropidococcus & 1 & & S Brasil & & & \\
\hline Marsileaceae & Regnellidium & 1 & Argentina & S Brasil & & & Uruguay \\
\hline
\end{tabular}




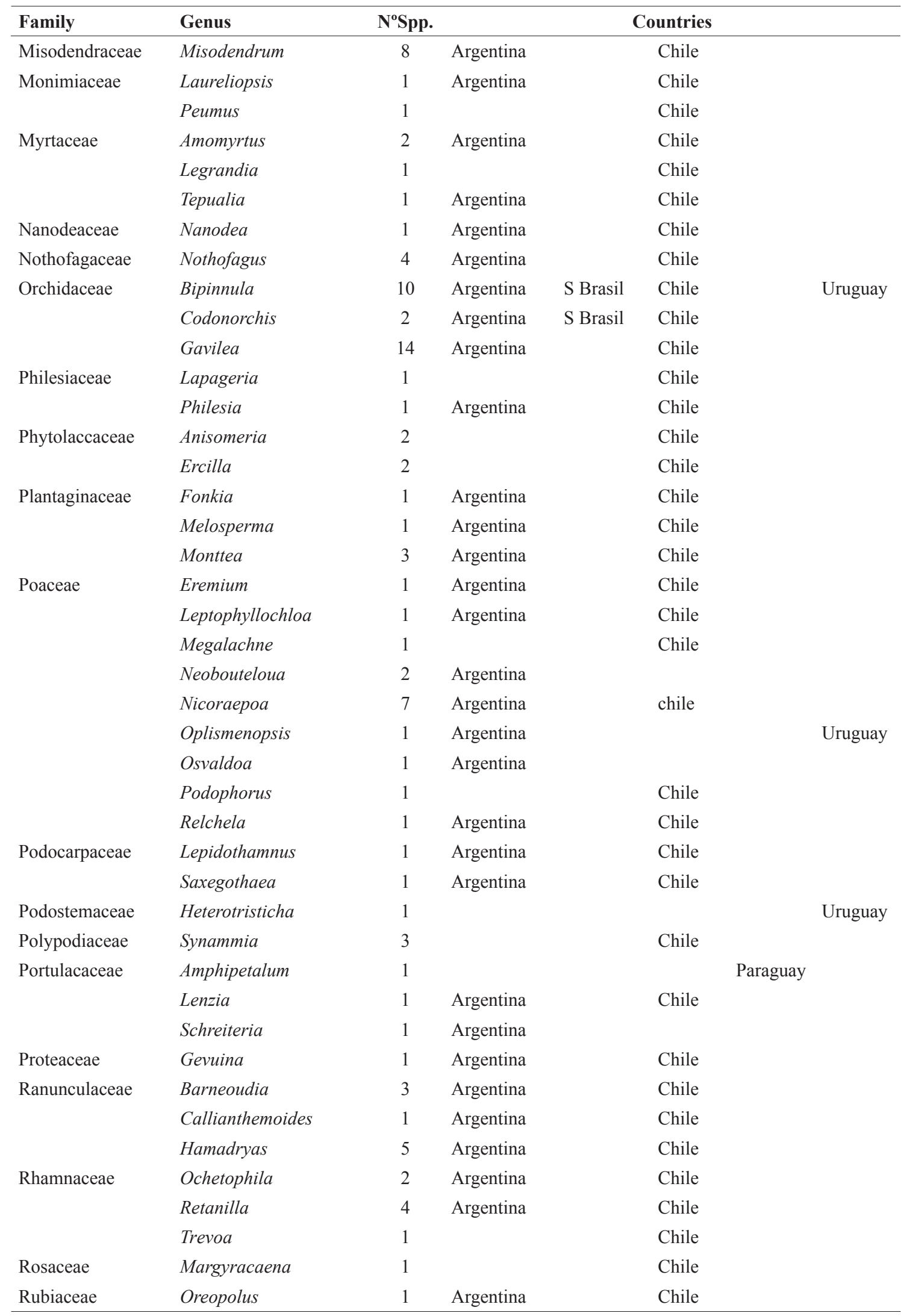




\begin{tabular}{|c|c|c|c|c|c|c|c|}
\hline Family & Genus & NoSpp. & & & Countries & & \\
\hline Rutaceae & Pitavia & 1 & & & Chile & & \\
\hline Rutaceae & Raulinoa & 1 & & S Brasil & & & \\
\hline Santalaceae & Myoschilos & 1 & Argentina & & Chile & & \\
\hline \multirow[t]{2}{*}{ Sapindaceae } & Bridgesia & 1 & & & Chile & & \\
\hline & Guindilia & 3 & Argentina & & Chile & & \\
\hline \multirow[t]{2}{*}{ Saxifragaceae } & Saxifragella & 1 & Argentina & & Chile & & \\
\hline & Saxifragodes & 1 & Argentina & & Chile & & \\
\hline \multirow[t]{9}{*}{ Solanaceae } & Benthamiella & 12 & Argentina & & Chile & & \\
\hline & Combera & 2 & Argentina & & Chile & & \\
\hline & Latua & 1 & & & Chile & & \\
\hline & Pantacantha & 1 & Argentina & & & & \\
\hline & Reyesia & 4 & Argentina & & Chile & & \\
\hline & Salpiglossis & 2 & Argentina & & Chile & & \\
\hline & Schizanthus & 14 & Argentina & & Chile & & \\
\hline & Sclerophylax & 14 & Argentina & & & Paraguay & Uruguay \\
\hline & Vestia & 1 & & & Chile & & \\
\hline \multirow[t]{2}{*}{ Tecophilaeaceae } & Conanthera & 5 & & & Chile & & \\
\hline & Tecophilaea & 2 & & & Chile & & \\
\hline Tribelaceae & Tribeles & 1 & Argentina & & Chile & & \\
\hline \multirow[t]{3}{*}{ Verbenaceae } & Dipyrena & 1 & Argentina & & & & \\
\hline & Neosparton & 4 & Argentina & & & & \\
\hline & Parodianthus & 2 & Argentina & & & & \\
\hline \multirow[t]{3}{*}{ Vivianiaceae } & Araeoandra & 1 & & & Chile & & \\
\hline & Cissarobryon & 1 & & & Chile & & \\
\hline & Viviania & 4 & Argentina & S Brasil & Chile & & \\
\hline \multirow[t]{3}{*}{ Zygophyllaceae } & Metharme & 1 & & & Chile & & \\
\hline & Pintoa & 1 & & & Chile & & \\
\hline & Plectrocarpa & 2 & Argentina & & & & \\
\hline
\end{tabular}

The Atlantic forest region, in the eastern extreme of Argentina and adjacent areas of Paraguay and southern Brazil, has high richness of families, genera and species, but there are few endemisms, with no records of endemic families, and just 9 genera that grow exclusively in the Paraná related forests of the Southern Cone: Quaternella Pederesen (Amaranthaceae), Eithea Ravenna (Amaryllidaceae), Criscia Katinas and Heterothalamulopsis Deble, A.S. Oliveira \& Marchiori (Asteraceae), Peregrina W.R. Anderson (Malpighiaceae), Calyptraemalva Krapov. and Tropidococcus Krapov. (Malvaceae), Amphipetalum Bacigalupo (Portulacaceae) and Raulinoa R.S. Cowan (Rutaceae).
In Chile, the region of the Chilean-Peruvian Desert includes aproximately 15 endemic genera, chiefly for the Cactaceae (Eriosyce Phil. and Thelocephala Y. Ito), Asteraceae (Gypothamnium Phil., Kieslingia Faúndez, Saldivia \& A.E. Martic. and Oxyphyllum Phil.), Alstroemeriaceae (Leontochir Phil.), Loasaceae (Huidobria Gay) and Zygophyllaceae (Metharme Phil. ex Engl. and Pintoa Gay), among others. A high number of endemic species also occurs in this region, including numerous species of Cryptantha G. Don and Heliotropium L. (Boraginaceae), Copiapoa Britton $\&$ Rose (Cactaceae) and Nolana L.f. (Solanaceae).

The Chilean mediterranean region comprises an important number of endemic taxa, with 
over 50 genera that are exclusive to this region or spreading to contiguous areas, for example Jubaea Kunth (Arecaceae), many genera of Amaryllidaceae (Gethyum Phil., Leucocoryne Lindl. and Speea Loes.), Asteraceae (Calopappus Meyen, Guynesomia Bonifacino \& Sancho, Leunisia Phil., Marticorenia Crisci, Moscharia Ruiz \& Pav., Pleocarphus D. Don and Podanthus Lag.), Bromeliaceae (Fascicularia Mez and Ochagavia Phil.), Monimiaceae (Peumus Molina), Tecophilaeaceae (Conanthera Ruiz \& Pav. and Tecophilaea Bertero ex Colla), Vivianiaceae (Araeoandra Lefor and Cissarobryon Kunze ex Poepp.), Francoaceae (Francoa Cav. and Tetilla DC.), Gomortegaceae (Gomortega Ruiz \& Pav.) and Zygophyllaceae (Pintoa). The number of endemic species is also outstanding.

For the Pampas region, there are 15 endemic genera, including representatives of Asteraceae (Asteropsis Less., Ianthopappus Roque \& D.J.N. Hind, Microgyne Less., Panphalea Lag. and Sommerfeltia Less.), Araceae (Mangonia Schott), Poaceae (Oplismenopsis Parodi and Osvaldoa), Amaryllidaceae (Beauverdia Herter and Ipheion Raf.), Apiaceae (Notiosciadium Speg.) and Apocynaceae (Rhyssostelma Decne.). The genus Sclerophylax Miers (Solanaceae) comprises 14 species chiefly from the Pampas with some extension into the Monte region.

The Monte includes many endemic genera, more than 20 exclusive from Argentina, such as Acanthocalycium Backeb., Denmoza Britton \& Rose, Setiechinopsis (Backeb.) de Haas and Tephrocactus Lem. (all Cactaceae), Ramorinoa Speg., Stenodrepanum Harms and Zuccagnia Cav. (Fabaceae), Dipyrena Hook. and Parodianthus Tronc. (Verbenaceae), Ixorhea Fenzl (Boraginaceae), Halosicyos Mart.Crov. (Cucurbitaceae), Plectrocarpa Gillies ex Hook. \& Arn. (Zygophyllaceae) and Tricomaria Hook. \& Arn. (Malpighiaceae). Genera found in the Monte sometimes extend into mediterranean Chile, such as Kurzamra Kuntze (Lamiaceae), Guindilia Hook. \& Arn. (Sapindaceae), Monttea Gay (Plantaginaceae) and Barneoudia Gay (Ranunculaceae).

Even taking into account that as one approaches higher latitudes the number of taxa diminishes, the number of endemic genera in the Patagonian region increases, with many representatives of Asteraceae (Ameghinoa Speg., Aylacophora Cabrera, Burkartia Crisci, Duseniella K. Schum., Lepidophyllum Cass. and Doniophyton
Wedd.), Brassicaceae (Chilocardamum O.E. Schulz, Delpinophytum Speg., Onuris Phil., Phlebolobium O.E. Schulz and Xerodraba Skottsb.), Cactaceae (Austrocactus Britton \& Rose, Maihuenia (F.C.A. Weber) K. Schum. and Pterocactus K. Schum.), Calyceraceae (Boopis Juss., Gamocarpha DC. and Nastanthus Miers), Fabaceae (Anarthrophyllum Benth.), Verbenaceae (Neosparton Griseb.), Solanaceae (Benthamiella Speg. and Pantacantha Speg.) and Malpighiaceae (Gallardoa Hicken). Genera present in this region and also in central Chile include Triptilion Ruiz \& Pav. (Asteraceae), Trichopetalum Lindl. (Laxmanniaceae), Melosperma Benth. (Plantaginaceae) and Retanilla (DC.) Brongn. (Rhamnaceae), Austrocactus and Maihuenia (Cactaceae). Amongst Patagonian genera also found in the Monte we have Lecanophora Speg. (Malvaceae) and Neosparton (Verbenaceae).

Only three genera are endemic from the Chaco: Lophocarpinia Burkart (Fabaceae), Bordasia Krapov. (Malvaceae) and Cordobia Nied. (Malpighiaceae), as this biome crosses over to Bolivia and the Central-Western area of Brazil (not included under this project).

Subantartic forests, within the temperatehumid Pacific region, include expressive numbers of endemic families and genera; however, the number of endemic species is not very high. The four endemic families from the region were indicated above; other families that have representatives in other places have here their unique presence in South America, as the Cupressaceae (with three endemic genera: Austrocedrus Florin \& Boutelje, Fitzroya Hook. f. ex Lindl. and Pilgerodendron Florin) and Restionaceae (Apodasmia B.G. Briggs \& L.A.S. Johnson). There are around 60 endemic genera in this region, among them the Juan Fernández Islands host 13, including an Arecaceae (Juania Drude), genera of Asteraceae (Centaurodendron Johow, Dendroseris D. Don, Yunquea Skottsb.), Boraginaceae (Selkirkia Hemsl.), Dicksoniaceae (Thyrsopteris Kunze), Lactoridaceae (Lactoris Phil.), Lamiaceae (Cuminia Colla), Myrtaceae (Nothomyrcia Kausel) and Poaceae (Megalachne Steud. and Podophorus Phil.). The continental zone has endemic genera that are exclusive from Chile or shared between Chile and Argentina of Asteraceae (Gamochaetopsis Anderb. \& S.E. Freire, Leptinella Cass., Leptocarpha DC. and Macrachaenium Hook.f.), Epacridaceae (Lebetanthus Endl.), Escalloniaceae (Valdivia 
Gay ex J. Rémy), Euphorbiaceae (Avellanita Phil.), Gesneriaceae (Asteranthera Hanst. and Sarmienta Ruiz \& Pav.), Iridaceae (Solenomelus Miers and Tapeinia Comm. ex Juss.), Lardizabalaceae (Boquila Decne. and Lardizabala Ruiz \& Pav.), Monimiaceae (Laureliopsis Schodde), Podocarpaceae (Lepidothamnus Phil. and Saxegothaea Lindl.), among others. Families Araucariaceae (Araucaria Juss.), Podocarpaceae (Podocarpus L'Her. ex Pers.), Thymelaeaceae (Ovidia Meisn. and Drapetes Lam.), Hymenophyllaceae (Hymenophyllum Sm.), Blechnaceae (Blechnum L.) and Winteraceae (Drimys J.R. Forst. \& G. Forst.) are disjunct between this region and the Atlantic Forest.

The analysis of the distribution of endemisms through the different biogeographic regions allows us to conclude that the number of endemic or exclusive families and genera is high in the Subantarctic forests, but there is a smaller number of endemic species in relation to other areas. Mediterranean Chile and deserts of Patagonia and Monte have a high number of endemic families, genera and species when compared with the totality of the analised flora, and such indices decrease as the latitude increases towards Patagonia, together with the general decrease in species richness of the vascular flora. The Chilean-Peruvian desert and adjacent high Andean and Puna areas in norhtern Argentina do not possess endemic families and the number of endemic genera is smaller, while the number of endemic species is outstanding. The amount of endemism diminishes markedly from west to east, with low numbers in the Chaco and Pampa, growing again, in terms of number of endemic species, in the Atlantic Forest from of eastern Paraguay, northeastern Argentina and southern Brazil.

\section{Introduced taxa}

A total of 19 introduced families are present in the Southern Cone, representing $6 \%$ of the total number of families in this region, namely: Aceraceae, Achariaceae, Agapanthaceae, Agavaceae, Aponogetonaceae, Asparagaceae, Asphodelaceae, Balsaminaceae, Cannabaceae, Caprifoliaceae, Dipsacaceae, Elaeagnaceae, Fumariaceae, Myoporaceae, Pinaceae, Resedaceae, Ruscaceae, Tamaricaceae and Zingiberaceae. The area counts with a total of 344 genera represented by introduced species $(12.84 \%)$ and a total of 1,129 introduced species ( $6.2 \%$ of the total of species found in the region). The family with the highest number of introduced genera is Asteraceae, with 54 genera, followed by Poaceae (42 genera), Brassicaceae (33), Fabaceae (22), Apiaceae (20), Lamiaceae (14) and Rosaceae (10) (Tab. 6).

The country where the highest record of introduced taxa was found is Argentina with 892, or $8.91 \%$ of the total number of species recorded for this country.

Table 6 - Southern Cone, introduced genera.

\begin{tabular}{|c|c|c|c|c|c|c|c|}
\hline Family & Genus & $\mathrm{N}^{0}$ Spp. & & & untries & & \\
\hline Acanthaceae & Hypoestes & 1 & & & & Paraguay & \\
\hline Aceraceae & Acer & 2 & Argentina & & & & \\
\hline Achariaceae & Hydnocarpus & 1 & & & & Paraguay & \\
\hline Agapanthaceae & Agapanthus & 1 & Argentina & & & & \\
\hline \multirow[t]{2}{*}{ Agavaceae } & Agave & 1 & Argentina & & & & \\
\hline & Yucca & 2 & Argentina & & & & \\
\hline \multirow[t]{4}{*}{ Aizoaceae } & Aptenia & 1 & Argentina & & & & \\
\hline & Cypselea & 1 & Argentina & & & & \\
\hline & Galenia & 1 & & & Chile & & \\
\hline & Sesuvium & 1 & Argentina & S Brasil & Chile & Paraguay & Uruguay \\
\hline Alismataceae & Alisma & 2 & Argentina & & Chile & & \\
\hline \multirow[t]{3}{*}{ Amaryllidaceae } & Allium & 6 & Argentina & & & & \\
\hline & Amaryllis & 1 & & S Brasil & Chile & Paraguay & \\
\hline & Brunsvigia & 1 & Argentina & & & & \\
\hline
\end{tabular}




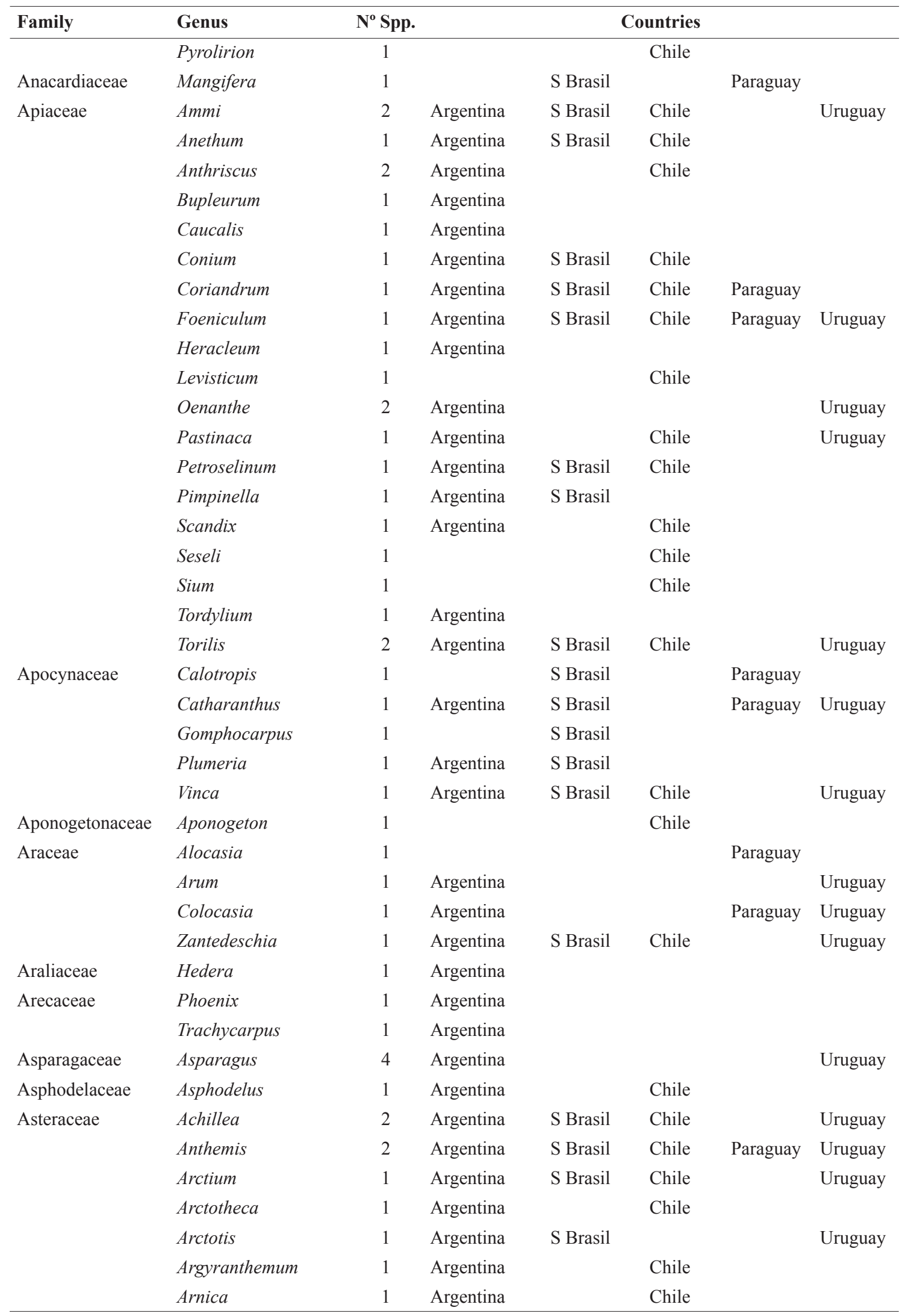




\begin{tabular}{|c|c|c|c|c|c|c|c|}
\hline Family & Genus & $\mathbf{N}^{0}$ Spp. & & & untries & & \\
\hline & Bellis & 1 & Argentina & & Chile & & \\
\hline & Calendula & 3 & Argentina & & Chile & & Uruguay \\
\hline & Carduus & 5 & Argentina & S Brasil & Chile & & Uruguay \\
\hline & Carthamus & 2 & Argentina & S Brasil & Chile & & Uruguay \\
\hline & Chondrilla & 1 & Argentina & & & & \\
\hline & Chrysanthemoides & 1 & & & Chile & & \\
\hline & Chrysanthemum & 2 & Argentina & & Chile & & Uruguay \\
\hline & Cichorium & 1 & Argentina & S Brasil & Chile & & Uruguay \\
\hline & Cirsium & 2 & Argentina & S Brasil & Chile & Paraguay & Uruguay \\
\hline & Cladanthus & 1 & Argentina & S Brasil & Chile & & Uruguay \\
\hline & Coleostephus & 1 & Argentina & S Brasil & Chile & & Uruguay \\
\hline & Crepis & 4 & Argentina & S Brasil & Chile & & Uruguay \\
\hline & Cynara & 1 & Argentina & S Brasil & Chile & & Uruguay \\
\hline & Dahlia & 1 & Argentina & & & & \\
\hline & Delairea & 1 & Argentina & & Chile & & Uruguay \\
\hline & Dyssodia & 1 & Argentina & & & & \\
\hline & Emilia & 1 & Argentina & S Brasil & & Paraguay & \\
\hline & Euryops & 1 & Argentina & & & & \\
\hline & Gazania & 1 & Argentina & & & & \\
\hline & Gynura & 1 & Argentina & & & & \\
\hline & Hedypnois & 1 & Argentina & & Chile & & Uruguay \\
\hline & Helianthus & 4 & Argentina & & & & Uruguay \\
\hline & Heterotheca & 1 & Argentina & & & & \\
\hline & Lactuca & 4 & Argentina & S Brasil & Chile & Paraguay & Uruguay \\
\hline & Lapsana & 1 & Argentina & S Brasil & Chile & & \\
\hline & Leontodon & 4 & Argentina & & Chile & & \\
\hline & Leucanthemum & 1 & Argentina & S Brasil & Chile & & Uruguay \\
\hline & Logfia & 1 & & & Chile & & \\
\hline & Malacothrix & 1 & Argentina & & Chile & & \\
\hline & Matricaria & 2 & Argentina & S Brasil & Chile & Paraguay & Uruguay \\
\hline & Microseris & 1 & & & Chile & & \\
\hline & Montanoa & 1 & Argentina & & & & \\
\hline & Onopordum & 2 & Argentina & S Brasil & Chile & & Uruguay \\
\hline & Picris & 1 & Argentina & & Chile & & Uruguay \\
\hline & Ratibida & 1 & Argentina & & & & \\
\hline & Rhaponticum & 1 & Argentina & & & & \\
\hline & Scolymus & 1 & Argentina & & Chile & & \\
\hline & Scorzonera & 1 & Argentina & & & & \\
\hline & Silybum & 1 & Argentina & S Brasil & Chile & & Uruguay \\
\hline & Sonchus & 4 & Argentina & S Brasil & Chile & Paraguay & Uruguay \\
\hline & Struchium & 1 & & S Brasil & & & \\
\hline & Tanacetum & 3 & Argentina & S Brasil & Chile & & Uruguay \\
\hline & Tithonia & 2 & Argentina & & & & \\
\hline
\end{tabular}




\begin{tabular}{|c|c|c|c|c|c|c|c|}
\hline Family & Genus & $\mathrm{N}^{0}$ Spp. & & & untries & & \\
\hline & Tolpis & 1 & & & Chile & & \\
\hline & Tragopogon & 3 & Argentina & & Chile & & \\
\hline & Tripleurospermum & 1 & Argentina & & Chile & & Uruguay \\
\hline & Urospermum & 1 & Argentina & & Chile & & Uruguay \\
\hline Athyriaceae & Deparia & 1 & Argentina & S Brasil & & & \\
\hline Balsaminaceae & Impatiens & 3 & Argentina & S Brasil & & & \\
\hline \multirow[t]{2}{*}{ Bignoniaceae } & Campsis & 1 & Argentina & & & & \\
\hline & Podranea & 1 & Argentina & S Brasil & & & \\
\hline \multirow[t]{8}{*}{ Boraginaceae } & Anchusa & 1 & Argentina & & & & \\
\hline & Asperugo & 1 & Argentina & & & & \\
\hline & Borago & 1 & Argentina & & Chile & & Uruguay \\
\hline & Echium & 1 & Argentina & & Chile & & \\
\hline & Lithospermum & 2 & Argentina & & & & Uruguay \\
\hline & Lycopsis & 1 & Argentina & & & & \\
\hline & Omphalodes & 1 & & & Chile & & \\
\hline & Symphytum & 1 & Argentina & & & & \\
\hline \multirow[t]{27}{*}{ Brassicaceae } & Aethionema & 1 & Argentina & & & & \\
\hline & Alliaria & 1 & Argentina & & & & \\
\hline & Alyssum & 1 & Argentina & & Chile & & \\
\hline & Arabidopsis & 1 & Argentina & & Chile & & Uruguay \\
\hline & Arabis & 1 & Argentina & & & & \\
\hline & Barbarea & 2 & Argentina & & Chile & & \\
\hline & Brassica & 5 & Argentina & & Chile & Paraguay & Uruguay \\
\hline & Cakile & 1 & Argentina & & & & Uruguay \\
\hline & Camelina & 3 & Argentina & & Chile & & Uruguay \\
\hline & Capsella & 1 & Argentina & S Brasil & Chile & & Uruguay \\
\hline & Chorispora & 1 & Argentina & & Chile & & \\
\hline & Cochlearia & 1 & Argentina & & & & \\
\hline & Diplotaxis & 3 & Argentina & & Chile & & Uruguay \\
\hline & Eruca & 1 & Argentina & & Chile & & \\
\hline & Erysimum & 2 & Argentina & & & & \\
\hline & Hesperis & 1 & Argentina & & Chile & & \\
\hline & Hirschfeldia & 1 & Argentina & & Chile & & Uruguay \\
\hline & Hornungia & 1 & Argentina & & Chile & & \\
\hline & Iberis & 1 & Argentina & & & & \\
\hline & Isatis & 1 & & & Chile & & \\
\hline & Lobularia & 1 & Argentina & & Chile & & Uruguay \\
\hline & Lunaria & 1 & Argentina & & & & \\
\hline & Malcolmia & 1 & Argentina & & & & \\
\hline & Matthiola & 1 & & & Chile & & \\
\hline & Nasturtium & 2 & Argentina & S Brasil & Chile & & Uruguay \\
\hline & Neslia & 1 & Argentina & & & & \\
\hline & Raphanus & 3 & Argentina & S Brasil & Chile & Paraguay & Uruguay \\
\hline
\end{tabular}




\begin{tabular}{|c|c|c|c|c|c|c|c|}
\hline Family & Genus & $\mathrm{N}^{0}$ Spp. & & & untries & & \\
\hline & Rapistrum & 1 & Argentina & S Brasil & Chile & Paraguay & Uruguay \\
\hline & Sinapis & 2 & Argentina & & Chile & Paraguay & \\
\hline & Sisymbrium & 7 & Argentina & S Brasil & Chile & & Uruguay \\
\hline & Strigosella & 1 & Argentina & & & & \\
\hline & Teesdalia & 1 & & & Chile & & \\
\hline & Thlaspi & 1 & Argentina & & Chile & & \\
\hline \multirow[t]{2}{*}{ Cactaceae } & Cylindropuntia & 1 & Argentina & & Chile & & \\
\hline & Nopalea & 1 & & & & Paraguay & \\
\hline \multirow[t]{2}{*}{ Campanulaceae } & Campanula & 1 & Argentina & & & & \\
\hline & Hippobroma & 1 & & S Brasil & & & \\
\hline Cannabaceae & Humulus & 2 & Argentina & & & & \\
\hline \multirow[t]{2}{*}{ Caprifoliaceae } & Lonicera & 1 & Argentina & S Brasil & & & Uruguay \\
\hline & Symphoricarpos & 1 & Argentina & & & & \\
\hline \multirow[t]{9}{*}{ Caryophyllaceae } & Agrostemma & 1 & Argentina & & Chile & & Uruguay \\
\hline & Dianthus & 2 & Argentina & & Chile & & \\
\hline & Herniaria & 2 & Argentina & & Chile & & \\
\hline & Holosteum & 1 & Argentina & & & & \\
\hline & Lychnis & 1 & & S Brasil & Chile & & \\
\hline & Petrorhagia & 3 & Argentina & & Chile & & \\
\hline & Saponaria & 1 & Argentina & & Chile & & Uruguay \\
\hline & Scleranthus & 1 & Argentina & & Chile & & Uruguay \\
\hline & Vaccaria & 1 & Argentina & & & & Uruguay \\
\hline \multirow[t]{6}{*}{ Chenopodiaceae } & Bassia & 2 & Argentina & & & & \\
\hline & Beta & 1 & Argentina & & Chile & & \\
\hline & Blitum & 1 & Argentina & & & & \\
\hline & Chenopodiastrum & 1 & Argentina & & Chile & & Uruguay \\
\hline & Cycloloma & 1 & Argentina & & & & \\
\hline & Salsola & 2 & Argentina & & Chile & & Uruguay \\
\hline Crassulaceae & Kalanchoe & 1 & Argentina & & & & \\
\hline \multirow[t]{4}{*}{ Cucurbitaceae } & Citrullus & 2 & Argentina & S Brasil & & Paraguay & \\
\hline & Cucumis & 1 & Argentina & S Brasil & & Paraguay & \\
\hline & Lagenaria & 1 & Argentina & S Brasil & & Paraguay & Uruguay \\
\hline & Luffa & 3 & Argentina & S Brasil & & Paraguay & \\
\hline Cupressaceae & Juniperus & 1 & Argentina & & & & \\
\hline \multirow[t]{3}{*}{ Dipsacaceae } & Dipsacus & 1 & Argentina & & & & Uruguay \\
\hline & Knautia & 2 & Argentina & & Chile & & \\
\hline & Scabiosa & 1 & Argentina & & Chile & & Uruguay \\
\hline Dryopteridaceae & Cyrtomium & 1 & Argentina & & & & \\
\hline Elaeagnaceae & Elaeagnus & 1 & Argentina & & & & \\
\hline \multirow[t]{4}{*}{ Euphorbiaceae } & Aleurites & 1 & Argentina & & & & \\
\hline & Breynia & 1 & & S Brasil & & & \\
\hline & Mercurialis & 1 & Argentina & & Chile & & \\
\hline & Ricinus & 1 & Argentina & S Brasil & Chile & Paraguay & Uruguay \\
\hline
\end{tabular}




\begin{tabular}{|c|c|c|c|c|c|c|c|}
\hline Family & Genus & $\mathbf{N}^{0}$ Spp. & & & untries & & \\
\hline & Vernicia & 1 & Argentina & & & & \\
\hline \multirow[t]{22}{*}{ Fabaceae } & Adenocarpus & 1 & & & & & Uruguay \\
\hline & Amorpha & 1 & Argentina & & & & Uruguay \\
\hline & Cajanus & 1 & & S Brasil & & Paraguay & \\
\hline & Colutea & 1 & Argentina & & & & \\
\hline & Coronilla & 1 & Argentina & & & & \\
\hline & Cytisus & 2 & Argentina & & Chile & & \\
\hline & Dipogon & 1 & & & Chile & & \\
\hline & Galega & 1 & Argentina & & Chile & & Uruguay \\
\hline & Genista & 1 & Argentina & & & & \\
\hline & Lablab & 1 & & & & & Uruguay \\
\hline & Laburnum & 1 & Argentina & & & & \\
\hline & Leucaena & 1 & Argentina & & & & \\
\hline & Medicago & 9 & Argentina & S Brasil & Chile & & Uruguay \\
\hline & Melilotus & 4 & Argentina & S Brasil & Chile & Paraguay & Uruguay \\
\hline & Neonotonia & 1 & Argentina & & & Paraguay & \\
\hline & Ononis & 1 & & & & & Uruguay \\
\hline & Psoralea & 1 & & & & & Uruguay \\
\hline & Robinia & 1 & Argentina & & Chile & & Uruguay \\
\hline & Spartium & 1 & Argentina & & Chile & & Uruguay \\
\hline & Sphaerophysa & 1 & Argentina & & & & \\
\hline & Trigonella & 1 & Argentina & & Chile & & \\
\hline & Ulex & 1 & Argentina & S Brasil & Chile & & Uruguay \\
\hline Fumariaceae & Fumaria & 7 & Argentina & & Chile & & Uruguay \\
\hline Gentianaceae & Blackstonia & 1 & Argentina & & & & Uruguay \\
\hline Gentianaceae & Irlbachia & 1 & & & & Paraguay & \\
\hline Hypoxidaceae & Molineria & 1 & Argentina & & & & \\
\hline \multirow[t]{9}{*}{ Iridaceae } & Belamcanda & 1 & & & & Paraguay & \\
\hline & Chasmanthe & 1 & Argentina & & & & \\
\hline & Crocosmia & 1 & Argentina & & Chile & & \\
\hline & Freesia & 2 & Argentina & & & & \\
\hline & Gladiolus & 2 & Argentina & & & & \\
\hline & Iris & 2 & Argentina & & Chile & & Uruguay \\
\hline & Romulea & 1 & & & Chile & & \\
\hline & Sparaxis & 2 & Argentina & & Chile & & \\
\hline & Watsonia & 1 & Argentina & & & & \\
\hline Juglandaceae & Carya & 1 & Argentina & & & & \\
\hline \multirow[t]{6}{*}{ Lamiaceae } & Ballota & 1 & Argentina & & & & Uruguay \\
\hline & Galeopsis & 1 & & & Chile & & \\
\hline & Glechoma & 1 & Argentina & & Chile & & \\
\hline & Lamium & 3 & Argentina & & Chile & & Uruguay \\
\hline & Leonotis & 1 & Argentina & S Brasil & & Paraguay & Uruguay \\
\hline & Leonurus & 2 & Argentina & S Brasil & & Paraguay & Uruguay \\
\hline
\end{tabular}




\begin{tabular}{|c|c|c|c|c|c|c|c|}
\hline Family & Genus & $\mathbf{N}^{\circ}$ Spp. & & & untries & & \\
\hline & Lycopus & 1 & & & Chile & & \\
\hline & Marrubium & 1 & Argentina & S Brasil & Chile & & Uruguay \\
\hline & Melissa & 1 & Argentina & & Chile & & Uruguay \\
\hline & Mentha & 4 & Argentina & S Brasil & Chile & Paraguay & Uruguay \\
\hline & Mesosphaerum & 1 & & S Brasil & & & \\
\hline & Molucella & 1 & Argentina & & Chile & & \\
\hline & Nepeta & 1 & Argentina & & & & \\
\hline & Prunella & 1 & Argentina & S Brasil & Chile & & Uruguay \\
\hline Lemnaceae & Landoltia & 1 & & & Chile & & \\
\hline Linderniaceae & Torenia & 1 & & S Brasil & & & \\
\hline Malvaceae & Alcea & 1 & Argentina & & & & \\
\hline & Gossypium & 1 & & & Chile & & \\
\hline & Malva & 7 & Argentina & S Brasil & Chile & Paraguay & Uruguay \\
\hline Meliaceae & Melia & 1 & Argentina & S Brasil & Chile & Paraguay & Uruguay \\
\hline Montiaceae & Claytonia & 1 & Argentina & & & & \\
\hline Moraceae & Broussonetia & 1 & Argentina & & & & \\
\hline Myoporaceae & Myoporum & 1 & Argentina & & Chile & & Uruguay \\
\hline Myrtaceae & Eucalyptus & 2 & Argentina & & & & \\
\hline Nymphaeaceae & Nelumbo & 1 & & & & Paraguay & \\
\hline Oleaceae & Fraxinus & 3 & Argentina & S Brasil & & & Uruguay \\
\hline & Ligustrum & 2 & Argentina & & & & Uruguay \\
\hline & Syringa & 1 & Argentina & & & & \\
\hline Papaveraceae & Chelidonium & 1 & Argentina & & & & \\
\hline & Eschscholzia & 1 & Argentina & & Chile & & \\
\hline & Glaucium & 1 & Argentina & & & & \\
\hline & Papaver & 5 & Argentina & & Chile & & Uruguay \\
\hline Phrymaceae & Mazus & 1 & Argentina & S Brasil & & & \\
\hline Pinaceae & Pinus & 3 & Argentina & & Chile & & \\
\hline & Pseudotsuga & 1 & Argentina & & & & \\
\hline Plantaginaceae & Antirrhinum & 2 & Argentina & & & & Uruguay \\
\hline & Cymbalaria & 1 & Argentina & & Chile & & Uruguay \\
\hline & Digitalis & 1 & Argentina & S Brasil & Chile & & Uruguay \\
\hline & Kickxia & 1 & Argentina & & Chile & & Uruguay \\
\hline & Linaria & 3 & Argentina & S Brasil & Chile & & Uruguay \\
\hline & Maurandya & 3 & Argentina & S Brasil & & & \\
\hline Poaceae & Agropyron & 1 & & & Chile & & \\
\hline & Aira & 4 & Argentina & S Brasil & Chile & & Uruguay \\
\hline & Apera & 1 & Argentina & & Chile & & \\
\hline & Arrhenatherum & 1 & Argentina & & Chile & & \\
\hline & Arundo & 1 & Argentina & S Brasil & Chile & & \\
\hline & Austrostipa & 1 & Argentina & & & & \\
\hline & Avena & 6 & Argentina & S Brasil & Chile & & Uruguay \\
\hline & Brachypodium & 2 & Argentina & & Chile & & Uruguay \\
\hline
\end{tabular}




\begin{tabular}{|c|c|c|c|c|c|c|c|}
\hline \multirow[t]{2}{*}{ Family } & \multirow{2}{*}{$\begin{array}{l}\text { Genus } \\
\text { Briza }\end{array}$} & \multirow{2}{*}{$\frac{\mathbf{N}^{0} \text { Spp. }}{3}$} & \multicolumn{5}{|c|}{ Countries } \\
\hline & & & Argentina & S Brasil & Chile & & Uruguay \\
\hline & Catapodium & 1 & Argentina & S Brasil & Chile & & Uruguay \\
\hline & Chrysopogon & 1 & Argentina & & & Paraguay & \\
\hline & Coix & 1 & Argentina & S Brasil & & Paraguay & \\
\hline & Corynephorus & 2 & Argentina & & Chile & & \\
\hline & Cymbopogon & 1 & Argentina & & Chile & & \\
\hline & Cynosurus & 2 & Argentina & & Chile & & Uruguay \\
\hline & Dactylis & 1 & Argentina & S Brasil & Chile & & Uruguay \\
\hline & Dactyloctenium & 1 & Argentina & S Brasil & & Paraguay & Uruguay \\
\hline & Dichanthium & 2 & Argentina & & & Paraguay & \\
\hline & Ehrharta & 1 & Argentina & & & & \\
\hline & Elytrigia & 1 & Argentina & & Chile & & \\
\hline & Gaudinia & 1 & Argentina & & & & Uruguay \\
\hline & Hackelochloa & 1 & Argentina & & & Paraguay & \\
\hline & Hainardia & 1 & Argentina & & Chile & & Uruguay \\
\hline & Hemarthria & 1 & Argentina & S Brasil & & Paraguay & Uruguay \\
\hline & Holcus & 1 & Argentina & S Brasil & Chile & & Uruguay \\
\hline & Lachnagrostis & 1 & Argentina & & Chile & & \\
\hline & Lagurus & 1 & Argentina & S Brasil & Chile & & Uruguay \\
\hline & Lamarckia & 1 & Argentina & & Chile & & \\
\hline & Leymus & 1 & Argentina & & Chile & & \\
\hline & Megathyrsus & 1 & Argentina & S Brasil & & Paraguay & Uruguay \\
\hline & Melinis & 2 & Argentina & S Brasil & & Paraguay & Uruguay \\
\hline & Miscanthus & 1 & & & Chile & & Uruguay \\
\hline & Moorochloa & 1 & Argentina & & & & \\
\hline & Parapholis & 2 & Argentina & & Chile & & Uruguay \\
\hline & Piptatherum & 1 & Argentina & & Chile & & \\
\hline & Rottboellia & 1 & Argentina & & & & \\
\hline & Schedonorus & 1 & Argentina & & & & \\
\hline & Schismus & 2 & Argentina & & Chile & & \\
\hline & Sclerochloa & 1 & Argentina & & & & \\
\hline & Taeniatherum & 1 & & & Chile & & \\
\hline & Themeda & 1 & Argentina & & & Paraguay & \\
\hline & Thinopyrum & 1 & Argentina & & & & \\
\hline \multirow[t]{4}{*}{ Polygonaceae } & Antigonon & 1 & Argentina & & & Paraguay & \\
\hline & Emex & 1 & Argentina & & Chile & & Uruguay \\
\hline & Eriogonum & 1 & Argentina & & & & \\
\hline & Fallopia & 1 & & & Chile & & \\
\hline Primulaceae & Centunculus & 1 & Argentina & S Brasil & Chile & Paraguay & Uruguay \\
\hline Proteaceae & Grevillea & 1 & Argentina & & & & \\
\hline \multirow[t]{2}{*}{ Ranunculaceae } & Aquilegia & 1 & Argentina & & Chile & & \\
\hline & Ceratocephalus & 1 & Argentina & & & & \\
\hline Resedaceae & Reseda & 4 & Argentina & & Chile & & \\
\hline
\end{tabular}




\begin{tabular}{|c|c|c|c|c|c|c|c|}
\hline \multirow{2}{*}{$\begin{array}{l}\text { Family } \\
\text { Rhamnaceae }\end{array}$} & \multirow{2}{*}{$\begin{array}{l}\text { Genus } \\
\text { Hovenia }\end{array}$} & \multirow{2}{*}{$\frac{\mathbf{N}^{\mathbf{0}} \text { Spp. }}{1}$} & \multicolumn{5}{|c|}{ Countries } \\
\hline & & & Argentina & S Brasil & & Paraguay & \\
\hline \multirow[t]{10}{*}{ Rosaceae } & Cotoneaster & 1 & Argentina & & & & \\
\hline & Crataegus & 1 & Argentina & & & & \\
\hline & Duchesnea & 1 & Argentina & S Brasil & Chile & & Uruguay \\
\hline & Eriobotrya & 1 & Argentina & & & & \\
\hline & Malus & 2 & Argentina & & & & \\
\hline & Pyracantha & 1 & Argentina & & & & \\
\hline & Rosa & 5 & Argentina & & Chile & & \\
\hline & Sanguisorba & 1 & Argentina & & Chile & & \\
\hline & Sorbus & 1 & Argentina & & & & \\
\hline & Spiraea & 2 & Argentina & S Brasil & Chile & & \\
\hline \multirow[t]{2}{*}{ Rubiaceae } & Rubia & 1 & Argentina & & Chile & & \\
\hline & Sherardia & 1 & Argentina & & Chile & & Uruguay \\
\hline Ruscaceae & Cordyline & 1 & Argentina & S Brasil & & Paraguay & Uruguay \\
\hline \multirow[t]{3}{*}{ Rutaceae } & Citrus & 1 & Argentina & & & Paraguay & \\
\hline & Poncirus & 1 & Argentina & & & & \\
\hline & Ruta & 1 & Argentina & & Chile & & \\
\hline \multirow[t]{2}{*}{ Scrophulariaceae } & Scrophularia & 1 & & & Chile & & \\
\hline & Verbascum & 3 & Argentina & S Brasil & Chile & Paraguay & Uruguay \\
\hline Simaroubaceae & Ailanthus & 1 & Argentina & & Chile & & Uruguay \\
\hline Solanaceae & Brugmansia & 1 & Argentina & S Brasil & & Paraguay & \\
\hline Tamaricaceae & Tamarix & 1 & Argentina & & & & \\
\hline Theaceae & Camellia & 1 & Argentina & & & & \\
\hline Urticaceae & Soleirolia & 1 & & & Chile & & \\
\hline Valerianaceae & Centranthus & 1 & Argentina & & & & \\
\hline \multirow[t]{2}{*}{ Vitaceae } & Parthenocissus & 2 & Argentina & & & & \\
\hline & Vitis & 1 & Argentina & & & & \\
\hline Zingiberaceae & Hedychium & 1 & Argentina & S Brasil & & Paraguay & \\
\hline
\end{tabular}

\section{Conclusion}

The information compiled by these projects has proved the importance and richness of the flora of the temperate and cold-temperate ecosystems studied, the unique elements that integrate them and their relevance in terms of biodiversity. Therefore, continued projects to catalogue and study the vascular plants of meridional South America play an important part in the contribution towards the World Flora and, in a more immediate objective, in their input of data both to GBIF (Global Biodiversity Information Facility) and to the Sistema Nacional de Datos Biológicos of Argentina.

It is important to highlight as a positive aspect of these projects the cooperation that was established at national and international level with similar projects in neighbouring countries of South America, such as the Flora de Chile (edited by C. Marticorena \& R. Rodríguez at the Universidad de Concepción), Flora of Paraguay (edited by L. Ramella \& P. Perret, at the Geneva Botánic Garden) and the Lista do Brasil (coordinated by R. Campostrini Forzza, at the Jardim Botanico do Rio de Janeiro), which include shared objectives and work methodology.

As a synthesis, the development of projects to study plant diversity, uniting the work of botanists with tools such as databases, remote access to digitised herbarium specimens and bibliography are extremely beneficial because they lead to:

- Increment in plant knowledge and its availability for conservation purposes, providing a 
source of trusted and standardized data regarding Latin American botanical collections.

- Reinforcement of the collaboration between botanical institutes in South America.

- Strenghtening of the institutional activities, facilitating the informatization and digitisation processes of their collections and considerably increasing their visibility.

- Possibility to train young people in botanical collection management and informatization processing, producing high quality data.

- Facilitate taxonomic work by providing nonrestricted access to digitised botanical collections together with additional information comprising descriptions, photographs, illustrations, etc.

Looking towards the future, the biggest challenge we face is to obtain a sustainable support, from both the economic and the human points of view. Floristic works are at a disadvantage when compared with other studies that are often punctual and have more immediate results in terms of time, and also by the preference given to researchers who publish their findings in high impact journals. This situation has brought to a diminishing number of botanists being dedicated to floristic and monographic works, likewise to the ongoing important tasks of collecting and identifying plants in general.

\section{Acknowledgements}

We wish to thank the editors of Rodriguésia and the external reviewers for their valuable contribution to improving this article. The project was funded by the National Council for Scientific and Technical Research of Argentina (CONICET, PIP 11229100100207) and the National Geographic Society, grant 9398-13.

\section{References}

Anton, A.M. \& Zuloaga, F.O. (eds.). 2001-2005. Flora Fanerogámica Argentina. Fascículos 75-95. Programa ProFlora (CONICET).

Anton, A.M. \& Zuloaga, F.O. (eds.). 2012a. Brassicaceae. Flora Argentina. Estudio Sigma, Buenos Aires. Vol. 8, pp. 1-273.

Anton, A.M. \& Zuloaga, F.O. (eds.). 2012b. Verbenaceae. Flora Argentina. Estudio Sigma, Buenos Aires. Vol. 14 , pp. 1-220.

Grande Allende, J.R. 2014. Novitates Agrostologicae, IV. Additional segregates from Panicum incertae sedis. Phytoneuron 22: 1-6.

Guerreiro, C. \& Rúgolo de Agrasar, Z.E. 2013. Two new species of Chusquea (Poaceae, Bambuseae) from northwestern Argentina. Systematic Botany 38: 390-397.

Hunziker, A.T. (ed.). 1994-2001. Flora Fanerogámica Argentina. Fascículos 1-74. Programa ProFlora (CONICET).

O’Leary, N. \& Múlgura, M.E. 2012. A taxonomic revision of the genus Phyla L. Annals of the Missouri Botanical Garden 98: 578-596.

Josse, C.; Navarro, G.; Comer, P.; Evans, R.; FaberLangendoen, D.; Fellows, M.; Kittel, G.; Menard, S.; Pyne, M.; Reid, M.; Schulz, K.; Snow, K. \& Teague, J. 2003. Ecological systems of Latin America and the Caribbean: a working classification of terrestrial systems. NatureServe, Arlington. 47p.

Tryon, R.M. \& Tryon, A.F. 1982. Ferns and allied plants, with special reference to Tropical America. SpringerVerlag, New York, Heidelberg, Berlin. 857p.

Zuloaga, F.O.; Belgrano, M.J. \& Anton, A.M. 2013 (eds.). Solanaceae. Flora Argentina. Estudio Sigma, Buenos Aires. Vol. 13, pp. 1-349.

Zuloaga, F.O.; Belgrano, M.J. \& Anton, A.M. 2014a (eds.). Asteraceae, Anthemideae-Gnaphalieae. Flora Argentina. Estudio Sigma, Buenos Aires. Vol. 7, pars 1, pp. 1-546.

Zuloaga, F. O.; Belgrano, M. J. \& Anton, A. M. 2014 b (eds.). Asteraceae, Senecioneae-Vernonieae. Flora Argentina. Estudio Sigma, Buenos Aires. Vol. 7, pars 3, pp. 1-306.

Zuloaga, F.O. \& Morrone, O. (eds.) 1996. Catálogo de las plantas vasculares de la República Argentina. I. Pteridophyta, Gymnospermae \& Angiospermae (Monocotyledoneae). Monographs in Systematic Botany from the Missouri Botanical Garden 60: 1-323.

Zuloaga, F.O. \& Morrone, O. (eds.). 1999. Catálogo de las plantas vasculares de la República Argentina. II. Angiospermae (Dicotyledoneae). Monographs in Systematic Botany from the Missouri Botanical Garden 64: 1-1269.

Zuloaga, F.O.; Morrone, O. \& Belgrano, M.J. (eds.) 2008. Catálogo de las plantas vasculares del Cono Sur (Argentina, Sur de Brasil, Chile, Paraguay y Uruguay). Monographs in Systematic Botany from the Missouri Botanical Garden 107: i-xcvi + 1-3348.

Zuloaga, F.O.; Nicora, E.G.; Rúgolo de Agrasar, Z.E.; Morrone, O.; Pensiero, J.F. \& Cialdella, A.M. 1994. Catálogo de la familia Poaceae en la República Argentina. Monographs in Systematic Botany from the Missouri Botanical Garden 47: 1-178.

Zuloaga, F.O.; Rúgolo, Z.E. \& Anton, A.M. (eds.). 2012. Flora Argentina. Gráficamente Ediciones, Córdoba. 3(1) (Poaceae, Aristidoideae-Panicoideae): 1-588; 3(2) (Poaceae, Pooideae): 1-523.

Zuloaga, F.O.; Belgrano, M.J. \& Anton, A.M. (eds.). 2015. Annonaceae (...). Flora Argentina. Estudio Sigma, Buenos Aires. Vol. 15, pp. 1-118. 